\title{
Circular Time Scale Yields a Recurrent Calculation of the Schrödinger Perturbation Energy
}

\author{
Stanisław Olszewski \\ Institute of Physical Chemistry, Polish Academy of Science, Warsaw, Poland \\ Email:olsz@ichf.edu.pl
}

How to cite this paper: Olszewski, S. (2018) Circular Time Scale Yields a Recurrent Calculation of the Schrödinger Perturbation Energy. Journal of Modern Physics, 9, 1491-1521.

https://doi.org/10.4236/jmp.2018.98093

Received: April 25, 2018

Accepted: July 9, 2018

Published: July 12, 2018

Copyright $\odot 2018$ by author and Scientific Research Publishing Inc. This work is licensed under the Creative Commons Attribution International License (CC BY 4.0).

http://creativecommons.org/licenses/by/4.0/

\begin{abstract}
An essential simplification of approach to the Schrödinger perturbation series for energy does hold when the perturbation events are arranged along a circular scale of time. The aim of the present paper is to demonstrate how such a scale of time leads to the recurrence calculation process of the Schrödinger energy terms belonging to an arbitrary perturbation order $N$. This process seems to have never been represented before. Only a non-degenerate quantum state and its perturbation due to the space-dependent potential are considered in the paper.
\end{abstract}

\section{Keywords}

Time, Its Circular Scale, Schrödinger Perturbation Formalism

\section{Introduction}

In science an identical result obtained in two different ways does not necessarily mean an effect of a secondary importance. An example is the Schrödinger perturbation formalism. In order to get it Schrödinger elaborated a special treatment of the inhomogeneous differential equations in course of which the energies and wave functions of a stationary quantum state perturbed by a time-independent potential could be calculated with the aid of the energies and wave functions representing the unperturbed quantum states of a given system [1]. Usually the unperturbed system was less complicated than a perturbed one, and the perturbation was limited to the potentials difference entering the perturbed and original state.

An effective formalism leading to the Schrödinger results is based usually on an iterative process; see e.g. [2]. When an original Hamiltonian $\hat{H}_{0}$ is 
perturbed by $\lambda \hat{H}^{\prime}$, we have to solve the time-independent Schrödinger equation given in the form

$$
\hat{H}^{\mathrm{per}} \psi^{\mathrm{per}}=E^{\mathrm{per}} \psi^{\text {per }},
$$

the problem is approached by a substitution

$$
\begin{gathered}
\hat{H}^{\text {per }}=\hat{H}_{0}+\lambda \hat{H}^{\prime}, \\
\psi^{\text {per }}=\psi_{0}+\lambda \psi_{1}+\lambda^{2} \psi_{2}+\cdots
\end{gathered}
$$

and

$$
E^{\text {per }}=E_{0}+\lambda E_{1}+\lambda^{2} E_{2}+\cdots
$$

The $\hat{H}_{0}, E_{0}$ and $\psi_{0}$ are respectively the unperturbed Hamiltonian, energy and wave function, whereas $\lambda \hat{H}^{\prime}, \lambda E_{1}, \lambda^{2} E_{2}, \cdots$ and $\lambda \psi_{1}, \lambda^{2} \psi_{2}, \cdots$ represent respectively the perturbed quantities. The solution of (4) can be obtained gradually for different powers of $\lambda$. In the next step the size of the parameter $\lambda$ is put equal to 1 ; see [2].

This rather tedious procedure does not apply time, which makes it similar to the original Schrödinger approach [1]. For $\lambda=1$ the notation of (4) is usually changed into the expression

$$
E^{\text {per }}-E_{0}=\Delta E_{1}+\Delta E_{2}+\Delta E_{3}+\cdots
$$

where $\Delta E_{1}, \Delta E_{2}, \Delta E_{3}, \cdots$ are the perturbation energies corresponding to the so-called perturbation orders $N$ equal respectively to $N=1, N=2, N=3$, etc.

The time entered the Schrödinger perturbation theory-limited to the stationary quantum states-with the development of diagrams introduced by Feynman [3] [4]. But these diagrams were based on a different kind of the time scale than applied in the present paper. In order to clarify the origin of a difference between the Feynman and present perturbation formalism, a step towards the time backround entering both methods seems to be of use.

\section{Physics of a Quantum System and the Notion of Time}

Both physical and philosophical features connected with the notion of time are combined systematically with scientific experience and observations of everyday life. A separate component of our view on time is provided by human imagination. In effect the idea of the time notion is extended-with a variable degree of certainty-from the atomic world to universe.

In fact time is a parameter the knowledge of which depends both on the properties of the examined object as well as the abilities possessed by an observer. If we limit our "universe" to a single hydrogen atom and the observer's ability to distinguish between the atomic nucleus and electron together with the possibility to estimate the size of a distance between these two objects, we can obtain two kinds of observations. One of them is created by assuming that a constant distant does hold between the nucleus and electron. This situation cannot serve to establish any notion of time because no change of the distance parameter can be detected and observed. However another situation is obtained when the 
distance between two particles changes systematically in a planar motion of the electron particle, which has its trajectory say along an ellipse. In this case the observer's measurements are spread along the interval length which is equal to a difference between the larger and smaller semiaxis of the ellipse. If the motion is perfectly periodic, any point observed within the interval length repeats after the same period of the motion time $T$.

In effect all time points accessible by observations are enclosed within the interval

$$
0<t<T \text {, }
$$

which repeats incessibly because no limit is imposed on the electron motion along the ellipse.

But a huge amount of everyday observations is evidently against the limit given in (5). In fact a finite amount of $T$ is replaced by infinity, so

$$
0<t<\infty \text {. }
$$

On the other hand, an analysis of the contemporary situation as an effect of an earlier situation combined with imagination implies the past events qualitatively separated from the present situation by a time interval which can be also of an infinite size. This gives the interval

$$
-\infty<t<\infty
$$

where $t=0$ can be assumed to be close to the present time.

The interval (7) encloses practically all possible events in nature but does not explain much what happens, will happen, or has happened, within (6) or (7). A characteristics of time is often expected to be obtained from physics. In fact we look for an objective method to define this character. Perhaps the best known result is given by the second law of thermodynamics which applies the notion of entropy and states that "later" means systematically a larger entropy than the entropy at an "earlier" time. An objection which can be raised here is connected mainly with the fact of applying the thermodynamics and entropy: these notions concern macroscopic systems built up regularly from a huge number of individual components.

But difficulties with a physical approach to time concern also the quantum domain. First the time intervals of numerous quantum processes are too short to be satisfactorily controlled on both the theoretical and experimental level. However opposite cases can be also considered. If an atom is in its lowest energy state, called also a ground state, and no external forces or collisions act on it, this atomic state can be preserved infinitely with no change. Therefore-according to the present state of our knowledge-no idea or scale of time can be applied in describing such an atom. However, a different situation is obtained when-at some moment-the atom is perturbed, for example by an action of an external field which can be chosen to be independent of time. If the time moment of inclusion of the perturbation potential is denoted by

$$
t=t_{b}
$$


at any time moment

$$
t>t_{b}
$$

the properties of the atom are changed in comparison to those possessed in (8). But we can assume that in effect of the action of the perturbation potential

$$
V^{\text {per }}
$$

till some

$$
t_{e} \gg t_{b},
$$

the atom will approach another stationary state. In effect of that the atom properties at

$$
t \gg t_{e}
$$

will be not much different than those possessed near

$$
t=t_{e} \text {. }
$$

In other words the atom behaves at (12) as an unchanged object equal to that obtained at (13), therefore the notion of time looses again its sense. But a question arises now how the time is going on between $t=t_{b}$ and $t=t_{e}$. An attempt to answer this question became a major subject of the paper.

The answer is obtained with the aid of an analysis of the events which accompany the perturbation process. According to Leibniz [5] [6] it is the sequence of events which is legitimate to provide us with a knowledge of the character of the time scale associated with a given process. In this case the problem of the size of the time intervals between subsequent events becomes of a secondary importance, but the accent is put on the properties (regularities) of the changes of the system exhibited in course of the time flow.

One of the aims of the present paper is to compare two scales of time applied to the case of the perturbation process. The first-based on a linear scale extended from the minus to plus infinity [see (6) and (7)] - was involved in the Feynman's approach to quantum mechanics [3] [4], another scale-of an essentially circular character-has been developed by the author [7]-[14]

\section{Feynman's Approach and Present Approach to the Schrödinger Perturbation Energy}

An essential difference between these two approaches is that in a majority of calculations postulated according to the Feynman's scheme of diagramsespecially for a large perturbation order $N$-there exists no reference between the energy terms provided by the Schrödinger perturbation theory and the Feynman diagrams. A reason of that is the fact that for large $N$ the number of the Feynman diagrams equal to

$$
P_{N}=(N-1) \text { ! }
$$

does exceed dramatically the number of the Schrödinger terms given by the formula [15] [16]: 


$$
S_{N}=\frac{(2 N-2) !}{N !(N-1) !}
$$

The ratio between $P_{N}$ and $S_{N}$ is

$$
\frac{P_{N}}{S_{N}}=\frac{N ![(N-1) !]^{2}}{(2 N-2) !} ;
$$

evidently it increases rapidly with $N$. For example for $N=20$ the ratio (16) attains the number of

$$
\frac{P_{20}}{S_{20}}=0.688 \times 10^{8}
$$

This means that in average about $0.7 \times 10^{8}$ results of the integration prescribed by the Feynman diagrams should be combined in order to obtain one Schrödinger energy term. The task seems to be complicated even with the use of computers.

On the other hand, an evident advantage of the circular time scale is that it can provide us with a one-to-one correspondence between the diagrams based on the scale and the Schrödinger energy terms. This facilitates enormously any development of the Schrödinger perturbation calculation and serves to control its results.

\section{Basic Characteristics of the Circular Scale of Time}

The first rule concerning diagrams of the present theory is that they can be classified according to the perturbation orders

$$
N=1,2,3,4, \cdots
$$

characteristic for the Schrödinger perturbation scheme. This means that the number of time points taken into account on any diagram belonging to $N$ is equal to $N$. But only one diagram for each $N$ is represented by $N$ uncontracted time points labelled successively by

$$
1,2,3,4, \cdots, N \text {. }
$$

From the number $N$ of time points entering any diagram the points

$$
1,2,3,4, \cdots=M=N-1
$$

should be taken into account in formation of contractions of the time points characteristic for that diagram. A reason for that limitation is due to the fact that the point $N$, which is considered as a beginning-end point of the scale, does not enter contractions.

The contractions can be simple, i.e. between two points of time, viz.

$$
\begin{gathered}
t_{1}: t_{2}=1: 2, \\
t_{1}: t_{3}=1: 3, \\
t_{1}: t_{4}=1: 4, \cdots
\end{gathered}
$$

or 


$$
\begin{gathered}
t_{2}: t_{3}=1: 3, \\
t_{2}: t_{4}=2: 4, \cdots
\end{gathered}
$$

or

$$
t_{3}: t_{4}=3: 4, \cdots,
$$

but also double contractions like

$$
\begin{aligned}
& t_{1}: t_{2}: t_{3}=1: 2: 3, \\
& t_{1}: t_{2}: t_{4}=1: 2: 4, \\
& t_{1}: t_{3}: t_{4}=1: 3: 4, \cdots,
\end{aligned}
$$

and still more extended contractions with more than three time points involved in them can exist.

Evidently the time points are arranged on the scale, as well as in contraction ensembles, according to their rise in time:

$$
t_{1}<t_{2}<t_{3}<t_{4}<\cdots
$$

There are also possible combined time-points contractions, for example

$$
t_{1}: t_{2} \cap t_{3}: t_{4}=1: 2 \bigcap 3: 4
$$

or

$$
t_{1}: t_{4} \cap t_{2}: t_{3}=1: 4 \bigcap 2: 3,
$$

which indicate a simultaneous presence of two different contractions. The rule, however, for formation of such combined sets of contractions is that the loops created by them on a diagram cannot cross (see e.g. [7]). This means that, for example, such contractions combination like

$$
t_{1}: t_{3} \cap t_{2}: t_{4}=1: 3 \cap 2: 4
$$

cannot exist.

Physically any contraction of the time points creates one or more loops of time supplementary to the main-single for a given diagram-loop of time. These supplementary loops will be called the side loops of time. They can be regularly represented by the energy perturbation terms of the order lower than the actually examined $N$. The main loop of time should contain the beginning-end point characteristic for any considered $N$. This special time point-as it is stated above-does not participate in contractions.

\section{Loops of Time and Schrödinger Perturbation Terms for Energy}

In this Section the loops of time obtained for the circular-scale diagrams are referred to the Schrödinger perturbation terms for energy. A single diagram without contractions is present for any $N$; for the case of $N=7$ such diagram is drawn on Figure 1. The energy term corresponding to this diagram is

$$
\langle V P V P V P V P V P V P V\rangle \text {. }
$$

The number of $V$ is 7, but the number of $P$ is 6 . 


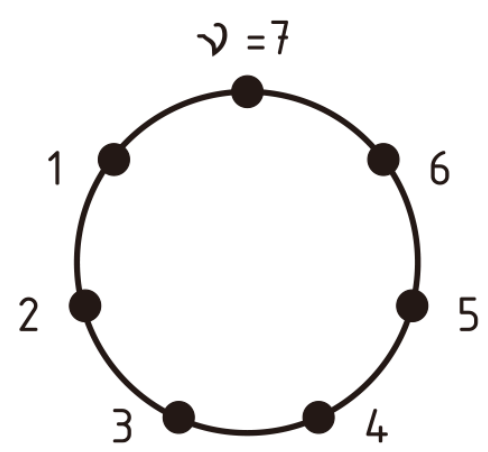

Figure 1. Diagram representing the energy term for the perturbation order $N=7$ having no contractions of the time points.

In general the symbol $V$ represents the matrix element

$$
\left\langle a\left|V^{\mathrm{per}}\right| b\right\rangle \text {. }
$$

The expression (25) begins with the matrix element (26) taken for $a=n$ and $b=p \neq n$ where $n$ is the index of quantum state submitted to perturbation. The next $V$ represent the matrix elements

$$
\left\langle p\left|V^{\text {per }}\right| q\right\rangle,\left\langle q\left|V^{\text {per }}\right| r\right\rangle,\left\langle r\left|V^{\text {per }}\right| s\right\rangle,\left\langle s\left|V^{\text {per }}\right| t\right\rangle,\left\langle t\left|V^{\text {per }}\right| u\right\rangle, \cdots
$$

and the last $V$ in $(25)$ is

$$
\left\langle u\left|V^{\mathrm{per}}\right| n\right\rangle .
$$

The successive symbols $P$ in (25) are respectively

$$
\begin{gathered}
P=\frac{1}{E_{n}-E_{p}}, \quad P=\frac{1}{E_{n}-E_{q}}, \quad P=\frac{1}{E_{n}-E_{r}}, \\
P=\frac{1}{E_{n}-E_{s}}, \quad P=\frac{1}{E_{n}-E_{t}}, \quad P=\frac{1}{E_{n}-E_{u}},
\end{gathered}
$$

and the whole expression (25) is a multiple sum performed over the quantum-state indices

$$
p, q, r, s, t, u, \cdots \neq n \text {. }
$$

The indices change from state 1 to infinity with the omission of state $n$ in each sum.

A contraction of two points, say

$$
p: q=1: 2,
$$

means creation of a side loop of time on the diagram of Figure 1 between the points 1 and 2; see Figure 2. In this case the perturbation term (25) changes into

$$
\left\langle V P^{2} V P V P V P V P V\right\rangle\langle V\rangle
$$

where

$$
P^{2}=\frac{1}{\left(E_{n}-E_{p}\right)^{2}}=\frac{1}{\left(E_{n}-E_{q}\right)^{2}}
$$




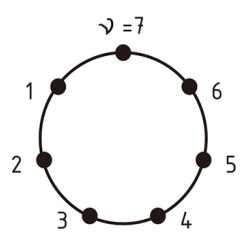

(1)

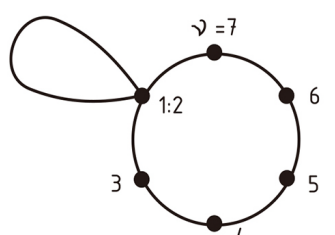

(2)

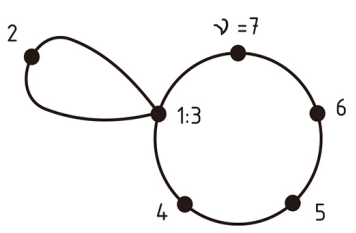

(3)

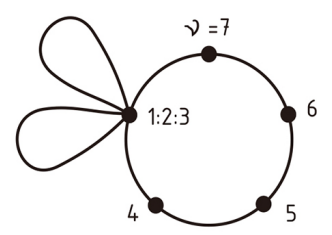

(4)

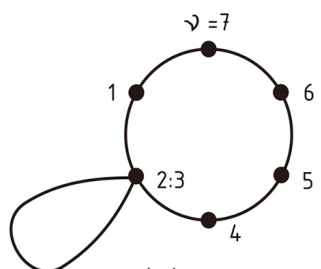

(5)

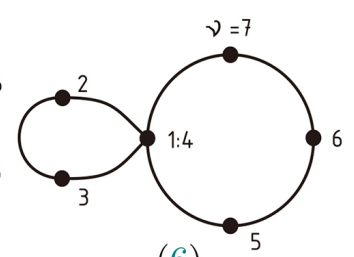

(6)

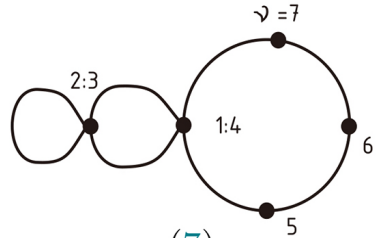

(7)

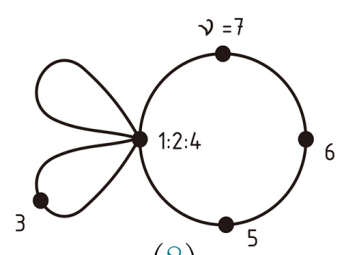

(8)

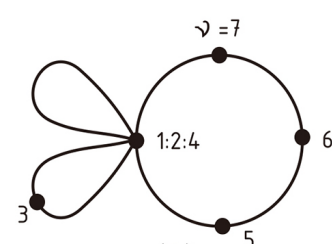

(9)

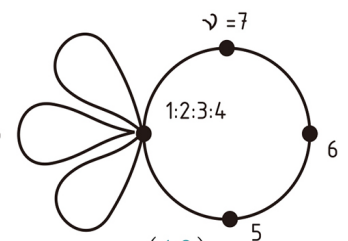

(10)
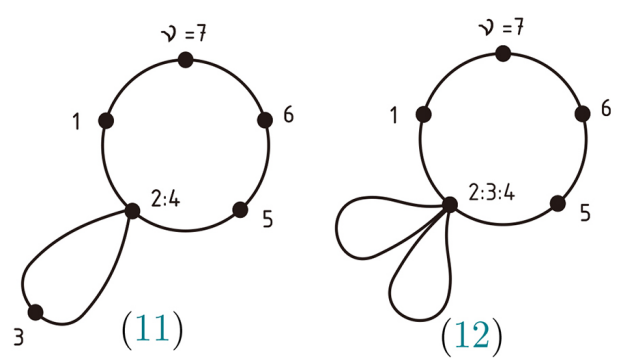

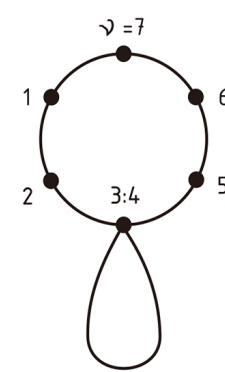

(13)

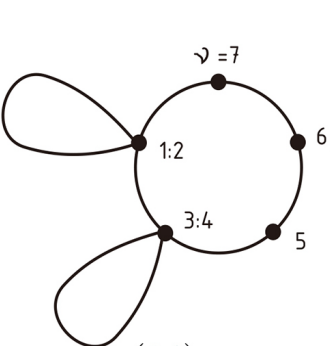

(14)

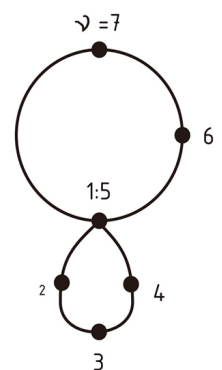

(15)

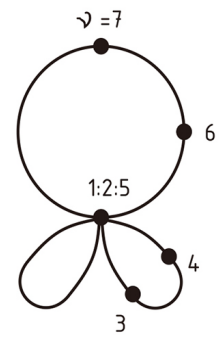

(16)

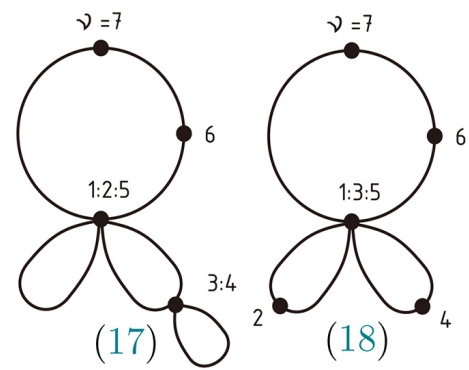

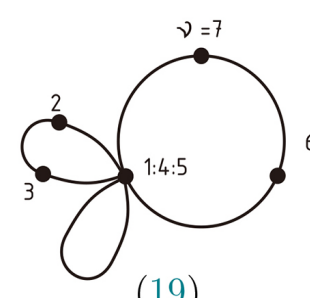

(19)

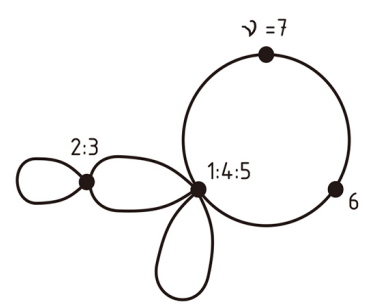

(20)

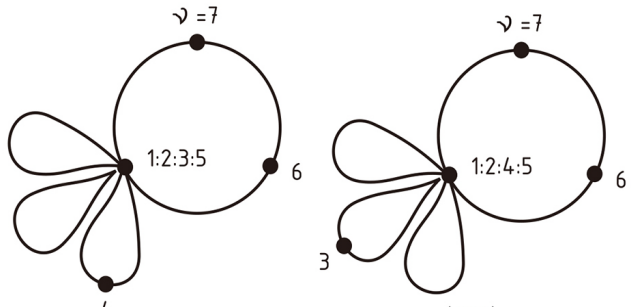

(21)

(22)

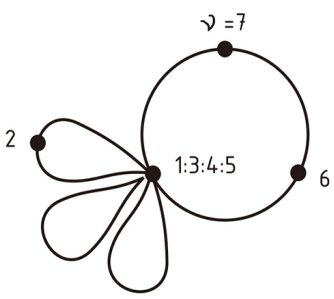

(23)

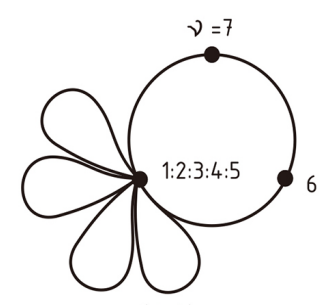

(24)

(a) 


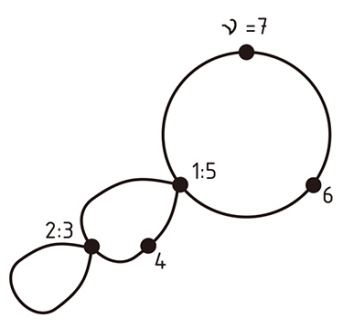

(25)

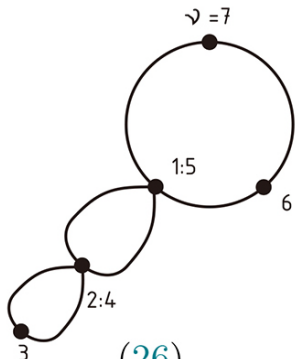

(26)

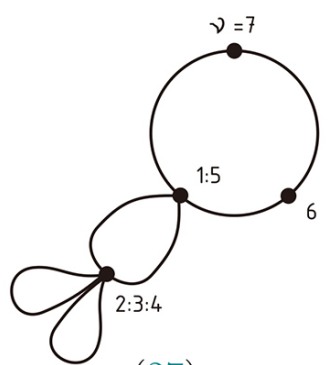

(27)

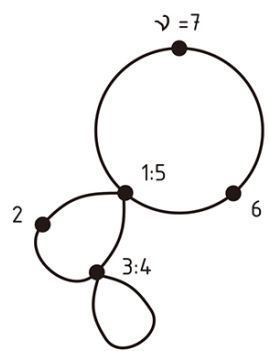

(28)

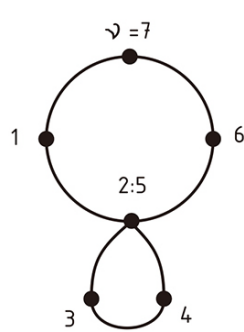

(29)

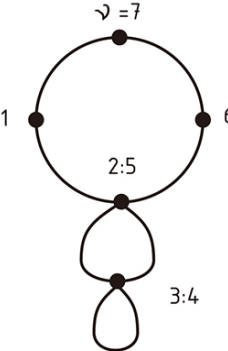

(30)

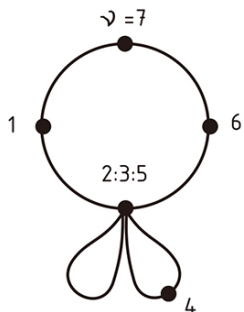

(31)

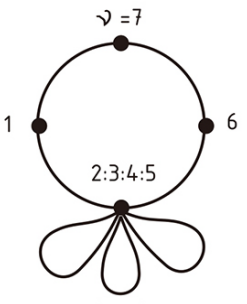

(32)

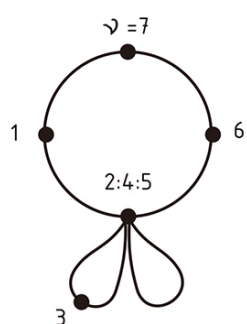

(33)

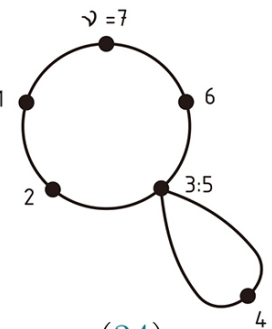

(34)

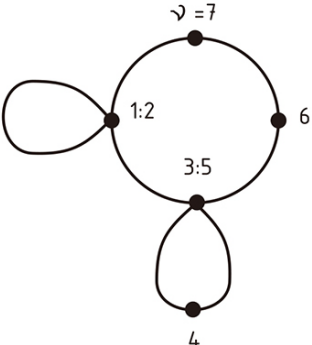

(35)

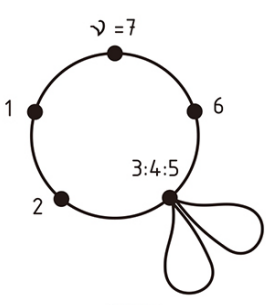

(36)

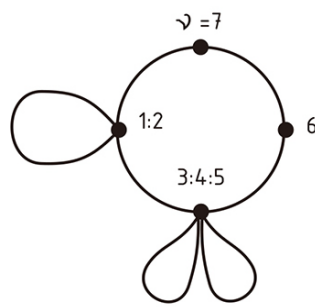

(37)

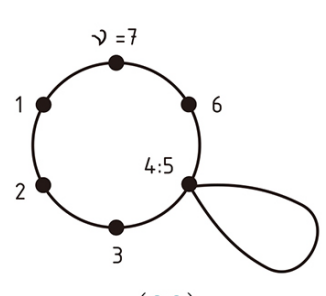

(38)

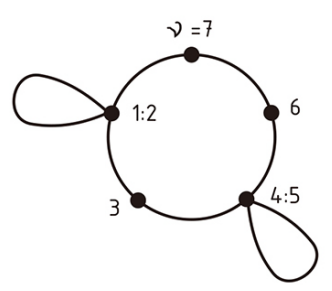

(39)

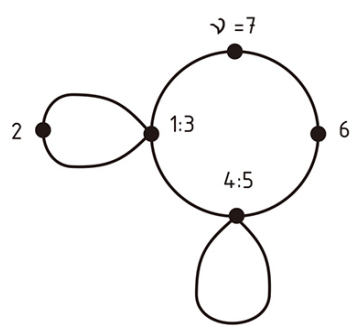

(40)

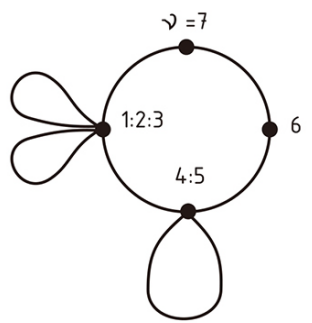

(41)

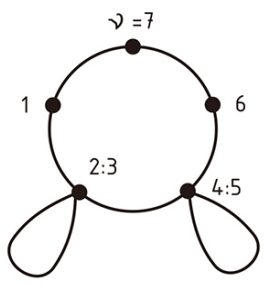

(42)

(b)

Figure 2. Diagrams representing the energy perturbation terms for $N=7$ obtained from a small modification of diagrams valid for the perturbation order $N=6$. The numbers below diagrams indicate the time contraction and energy term given in Table 1. 
and

$$
\langle V\rangle=\Delta E_{1}
$$

which is the first-order perturbation energy.

Contractions $2: 3,3: 4,4: 5$ and $5: 6$ give respectively the perturbation terms:

$$
\left\langle V P V P^{2} V P V P V P V\right\rangle\langle V\rangle
$$

with

$$
P^{2}=\frac{1}{\left(E_{n}-E_{q}\right)^{2}}=\frac{1}{\left(E_{n}-E_{r}\right)^{2}},
$$

the term

$$
\left\langle V P V P V P^{2} V P V P V\right\rangle\langle V\rangle
$$

with

$$
P^{2}=\frac{1}{\left(E_{n}-E_{r}\right)^{2}}=\frac{1}{\left(E_{n}-E_{s}\right)^{2}},
$$

the term

$$
\left\langle V P V P V P V P^{2} V P V\right\rangle\langle V\rangle
$$

with

$$
P^{2}=\frac{1}{\left(E_{n}-E_{s}\right)^{2}}=\frac{1}{\left(E_{n}-E_{t}\right)^{2}},
$$

the term

$$
\left\langle V P V P V P V P V P^{2} V\right\rangle\langle V\rangle
$$

with

$$
P^{2}=\frac{1}{\left(E_{n}-E_{t}\right)^{2}}=\frac{1}{\left(E_{n}-E_{u}\right)^{2}} .
$$

The summations entering (33)-(36) are extended respectively over

$$
\begin{aligned}
& p, q=r, s, t, u \neq n, \\
& p, q, r=s, t, u \neq n, \\
& p, q, r, s=t, u \neq n, \\
& p, q, r, s, t=u \neq n .
\end{aligned}
$$

Similar notation applies for other contractions than presented above. For example a double contraction

$$
p: q: r=1: 2: 3
$$

gives for the energy term coming from the main loop the expression

$$
\left\langle V P^{3} V P V P V P V\right\rangle(\langle V\rangle)^{2} .
$$

The term 


$$
(\langle V\rangle)^{2}=\left(\Delta E_{1}\right)^{2}
$$

is due to the side loops 1:2 and 2:3 separately. The term due to the main loop is represented by

$$
\left\langle V P^{3} V P V P V P V\right\rangle
$$

where

$$
P^{3}=\frac{1}{\left(E_{n}-E_{p}\right)^{3}}=\frac{1}{\left(E_{n}-E_{q}\right)^{3}}=\frac{1}{\left(E_{n}-E_{r}\right)^{3}} .
$$

Three other $P$ present in (40) are those given in (28) and summations concern the states

$$
s, t, u \text {. }
$$

In total the summations entering the brackets in (40) are extended over the states

$$
p=q=r, s, t, u \neq n .
$$

It is easy to prolongate the above notation to other contractions of the time points than considered between (30) and (43).

The sign of a perturbation term is dictated by the number of the bracket pairs which enter that term. For an odd number of the bracket pairs [see (25), (38)] the whole energy term should be taken with a positive sign, for an even number of the bracket pairs [see (31), (33)-(36)] the perturbation term should be taken with a minus sign.

\section{Recurrence Procedure for Calculating the Schrödinger Perturbation Terms Belonging to Arbitrary $N$}

It seems that the best way to represent this procedure is to apply it to an example. A particular task let be to derive the perturbation terms belonging to $N=7$ from the terms belonging to

$$
N<7 \text {. }
$$

The energy perturbation terms corresponding to $N$ entering (44) are briefly derived and given in Appendix. A question which can arise may be how similar terms should be calculated in the case of $N=7$.

The choice of $N=7$ means that a new free time point on the scale which can be submitted to contractions is

$$
N-1=6 .
$$

This implies that $S_{6}=42$ terms belonging to $N=6$ should be modified in order to take into account the presence of a free time point 6 absent in the case of $N=6$. In practice this means that all contributions coming to energy from the main loop of time for $N=6$ can be made valid also for $N=7$ on condition-at the end of any bracket term corresponding to the mentioned main loop of time-the product 
is added; see Table 1. The diagrams corresponding to the energy terms obtained in the way outlined below (45) are presented in Figure 2. This reduces the number of unknown terms for $N=7$, namely

$$
S_{7}=132,
$$

to

$$
S_{7}-S_{6}=132-42=90 .
$$

But the presence of point 6 on the time scale implies that this point has also to participate in contractions. The number of these contractions is obtained when contraction

$$
1: 6
$$

together with all admissible contractions of the time points on a circular scale between 1 and 6, are taken into account. The contraction (49) and its energy terms are

$$
1: 6 \rightarrow-\left\langle V P^{2} V\right\rangle \Delta E_{5} .
$$

This contraction provides us with contribution equal to 14 Schrödinger energy terms because the number of terms in $\Delta E_{5}$ is $S_{5}=14$. In the next step we obtain 5 terms, viz.

$$
1: 2: 6 \rightarrow\left\langle V P^{3} V\right\rangle \Delta E_{1} \Delta E_{4},
$$

because of $S_{1}=1$ and $S_{4}=5$. Two perturbation terms are given by

$$
1: 3: 6 \rightarrow\left\langle V P^{3} V\right\rangle \Delta E_{2} \Delta E_{3},
$$

because of $S_{2}=1$ and $S_{3}=2$; the same number of terms holds for contraction

$$
1: 4: 6 \rightarrow\left\langle V P^{3} V\right\rangle \Delta E_{3} \Delta E_{2} .
$$

Contraction

$$
1: 5: 6 \rightarrow\left\langle V P^{3} V\right\rangle \Delta E_{4} \Delta E_{1}
$$

gives $S_{4}=5$ terms since it is by symmetry similar to (51);

$$
1: 2: 3: 6 \rightarrow-\left\langle V P^{4} V\right\rangle\left(\Delta E_{1}\right)^{2} \Delta E_{3}
$$

gives two terms because of $S_{3}=2$;

$$
1: 2: 4: 6 \rightarrow-\left\langle V P^{4} V\right\rangle \Delta E_{1}\left(\Delta E_{2}\right)^{2}
$$

is a one-term contraction $\left(S_{1}=S_{2}=1\right)$;

$$
1: 2: 5: 6 \rightarrow-\left\langle V P^{4} V\right\rangle \Delta E_{1} \Delta E_{3} \Delta E_{1}
$$

gives two terms because of $S_{3}=2$;

$$
1: 3: 4: 6 \rightarrow-\left\langle V P^{4} V\right\rangle \Delta E_{2} \Delta E_{1} \Delta E_{2}
$$

is a one-term contraction;

$$
1: 3: 5: 6 \rightarrow-\left\langle V P^{4} V\right\rangle\left(\Delta E_{2}\right)^{2} \Delta E_{1}
$$


Table 1. Energy perturbation terms belonging to $N=7$ obtained by adding the product $P V$ from (46) into the main bracket terms entering the perturbation energy for $N=6$. The terms 15, 25, 26, 27 and 28 can combine into $-\left\langle V P^{2} V P V\right\rangle \Delta E_{4}$; the terms 6 and 7, 16 and 17,19 and 20, and 29 and 30 combine into four terms containing the factor of $\Delta E_{3}$.

\begin{tabular}{|c|c|c|}
\hline & contraction & energy term \\
\hline 1 & no contraction & $\langle V P V P V P V P V P V P V\rangle$ \\
\hline 2 & $1: 2$ & $-\left\langle V P^{2} V P V P V P V P V\right\rangle \Delta E_{1}$ \\
\hline 3 & $1: 3$ & $-\left\langle V P^{2} V P V P V P V\right\rangle \Delta E_{2}$ \\
\hline 4 & $1: 2: 3$ & $\left\langle V P^{3} V P V P V P V\right\rangle\left(\Delta E_{1}\right)^{2}$ \\
\hline 5 & $2: 3$ & $-\left\langle V P V P^{2} V P V P V P V\right\rangle \Delta E_{1}$ \\
\hline 6 & $1: 4$ & \multirow{2}{*}{$-\left\langle V P^{2} V P V P V\right\rangle \Delta E_{3}$} \\
\hline 7 & $1: 4 \cap 2: 3$ & \\
\hline 8 & $1: 2: 4$ & $\left\langle V P^{3} V P V P V\right\rangle \Delta E_{1} \Delta E_{2}$ \\
\hline 9 & $1: 3: 4$ & $\left\langle V P^{3} V P V P V\right\rangle \Delta E_{2} \Delta E_{1}$ \\
\hline 10 & $1: 2: 3: 4$ & $-\left\langle V P^{4} V P V P V\right\rangle\left(\Delta E_{1}\right)^{3}$ \\
\hline 11 & $2: 4$ & $-\left\langle V P V P^{2} V P V P V\right\rangle \Delta E_{2}$ \\
\hline 12 & $2: 3: 4$ & $\left\langle V P V P^{3} V P V P V\right\rangle\left(\Delta E_{1}\right)^{2}$ \\
\hline 13 & $3: 4$ & $-\left\langle V P V P V P^{2} V P V P V\right\rangle \Delta E_{1}$ \\
\hline 14 & $1: 2 \cap 3: 4$ & $\left\langle V P^{2} V P^{2} V P V P V\right\rangle\left(\Delta E_{1}\right)^{2}$ \\
\hline 15 & $1: 5$ & $-\left\langle V P^{2} V P V V P V P V P V\right\rangle$ \\
\hline 16 & $1: 2: 5$ & \multirow{2}{*}{$\left\langle V P^{3} V P V\right\rangle \Delta E_{1} \Delta E_{3}$} \\
\hline 17 & $1: 2: 5 \cap 3: 4$ & \\
\hline 18 & $1: 3: 5$ & $\left\langle V P^{3} V P V\right\rangle\left(\Delta E_{2}\right)^{2}$ \\
\hline 19 & $1: 4: 5$ & \multirow{2}{*}{$\left\langle V P^{3} V P V\right\rangle \Delta E_{3} \Delta E_{1}$} \\
\hline 20 & $1: 4: 5 \cap 2: 3$ & \\
\hline 21 & $1: 2: 3: 5$ & $-\left\langle V P^{4} V P V\right\rangle\left(\Delta E_{1}\right)^{2} \Delta E_{2}$ \\
\hline 22 & $1: 2: 4: 5$ & $-\left\langle V P^{4} V P V\right\rangle \Delta E_{1} \Delta E_{2} \Delta E_{1}$ \\
\hline 23 & $1: 3: 4: 5$ & $-\left\langle V P^{4} V P V\right\rangle \Delta E_{2}\left(\Delta E_{1}\right)^{2}$ \\
\hline 24 & $1: 2: 3: 4: 5$ & $\left\langle V P^{5} V P V\right\rangle\left(\Delta E_{1}\right)^{4}$ \\
\hline 25 & $1: 5 \cap 2: 3$ & $\left\langle V P^{2} V P V\right\rangle\left\langle V P^{2} V P V\right\rangle \Delta E_{1}$ \\
\hline 26 & $1: 5 \cap 2: 4$ & $\left\langle V P^{2} V P V\right\rangle\left\langle V P^{2} V\right\rangle \Delta E_{2}$ \\
\hline 27 & $1: 5 \cap 2: 3: 4$ & $\left\langle V P^{2} V P V\right\rangle\left\langle V P^{3} V\right\rangle\left(\Delta E_{1}\right)^{2}$ \\
\hline 28 & $1: 5 \cap 3: 4$ & $\left\langle V P^{2} V P V\right\rangle\left\langle V P V P^{2} V\right\rangle \Delta E_{1}$ \\
\hline 29 & $2: 5$ & \multirow{2}{*}{$-\left\langle V P V P^{2} V P V\right\rangle \Delta E_{3}$} \\
\hline 30 & $2: 5 \cap 3: 4$ & \\
\hline 31 & $2: 3: 5$ & $\left\langle V P V P^{3} V P V\right\rangle \Delta E_{1} \Delta E_{2}$ \\
\hline 32 & $2: 3: 4: 5$ & $-\left\langle V P V P^{4} V P V\right\rangle\left(\Delta E_{1}\right)^{3}$ \\
\hline 33 & $2: 4: 5$ & $\left\langle V P V P^{3} V P V\right\rangle \Delta E_{2} \Delta E_{1}$ \\
\hline
\end{tabular}




\section{Continued}

\begin{tabular}{ccc}
\hline 34 & $3: 5$ & $-\left\langle V P V P V P^{2} V P V\right\rangle \Delta E_{2}$ \\
35 & $1: 2 \cap 3: 5$ & $\left\langle V P^{2} V P^{2} V P V\right\rangle \Delta E_{1} \Delta E_{2}$ \\
36 & $3: 4: 5$ & $\left\langle V P V P V P^{3} V P V\right\rangle\left(\Delta E_{1}\right)^{2}$ \\
37 & $1: 2 \cap 3: 4: 5$ & $-\left\langle V P^{2} V P^{3} V P V\right\rangle\left(\Delta E_{1}\right)^{3}$ \\
38 & $4: 5$ & $-\left\langle V P V P V P V P^{2} V P V\right\rangle \Delta E_{1}$ \\
39 & $1: 2 \cap 4: 5$ & $\left\langle V P^{2} V P V P^{2} V P V\right\rangle\left(\Delta E_{1}\right)^{2}$ \\
40 & $1: 3 \cap 4: 5$ & $\left\langle V P^{2} V P^{2} V P V\right\rangle \Delta E_{2} \Delta E_{1}$ \\
41 & $1: 2: 3 \cap 4: 5$ & $-\left\langle V P^{3} V P^{2} V P V\right\rangle\left(\Delta E_{1}\right)^{3}$ \\
42 & $2: 3 \cap 4: 5$ & $\left\langle V P V P^{2} V P^{2} V P V\right\rangle\left(\Delta E_{1}\right)^{2}$ \\
\hline
\end{tabular}

gives also one term;

$$
1: 4: 5: 6 \rightarrow-\left\langle V P^{4} V\right\rangle \Delta E_{3}\left(\Delta E_{1}\right)^{2}
$$

is a two-terms contraction symmetrical to (55). The remaining one-term contractions joining points 1 and 6 are

$$
\begin{gathered}
1: 2: 3: 4: 6 \rightarrow\left\langle V P^{5} V\right\rangle\left(\Delta E_{1}\right)^{3} \Delta E_{2}, \\
1: 2: 3: 5: 6 \rightarrow\left\langle V P^{5} V\right\rangle\left(\Delta E_{1}\right)^{2} \Delta E_{2} \Delta E_{1}, \\
1: 2: 4: 5: 6 \rightarrow\left\langle V P^{5} V\right\rangle \Delta E_{1} \Delta E_{2}\left(\Delta E_{1}\right)^{2}, \\
1: 3: 4: 5: 6 \rightarrow\left\langle V P^{5} V\right\rangle \Delta E_{2}\left(\Delta E_{1}\right)^{3}, \\
1: 2: 3: 4: 5: 6 \rightarrow-\left\langle V P^{6} V\right\rangle\left(\Delta E_{1}\right)^{5} .
\end{gathered}
$$

In total we obtain from (50)-(65) the number of terms connected with the interaction between the time points 1 and 6 equal to:

$$
14+5+2+2+5+2+1+2+1+1+2+1+1+1+1+1=42 .
$$

In fact this is a number of the Schrödinger energy terms equal to

$$
S_{6}=42 \text {. }
$$

The diagrams corresponding to the terms obtained in (66), or (67), are represented in Figure 3. The calculation of the energy terms corresponding to diagrams entering (67) reduces the unknown number of the energy terms for $N$ $=7$ to

$$
S_{7}-2 \times S_{6}=132-84=48 \text {. }
$$

In order to present the terms (68)-see Figure 4-we take into account that the "interaction" of the time point 6 with point 1 can be extended by the "interaction" of point 2 with 6 in the absence of the interaction with point 1 . This provides us with contraction

$$
2: 6 \rightarrow-\left\langle V P V P^{2} V\right\rangle \Delta E_{4}
$$

because of $S_{4}=5$ this formula contains five Schrödinger terms. 


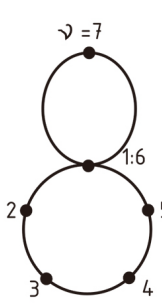

(50)

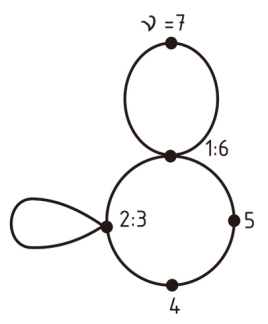

(50)-a

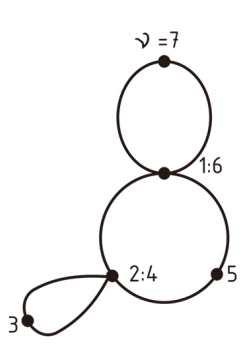

(50)-b

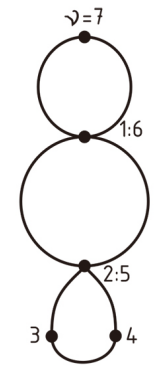

(50)-c

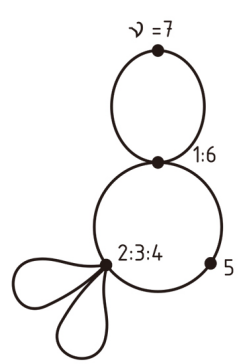

(50)-d

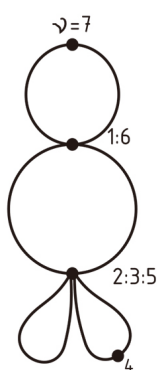

(50)-e

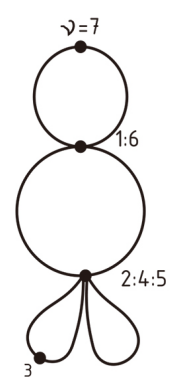

(50)-f

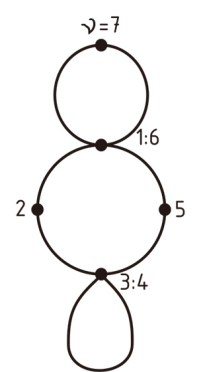

(50)-g

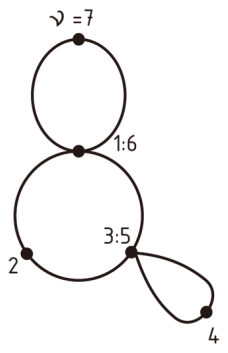

(50)-h

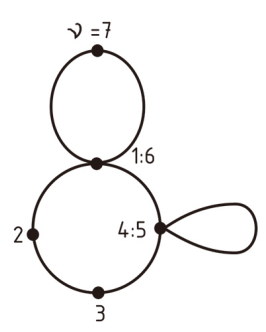

(50)-i

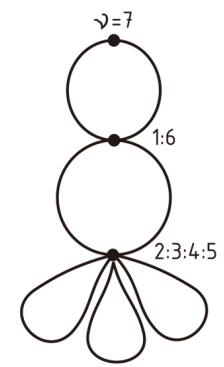

(50)-j

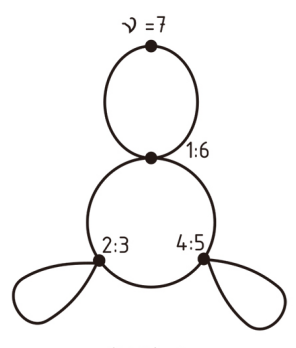

(50)-k

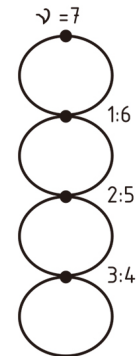

(50)-1

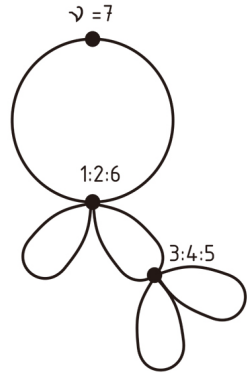

(51)-b

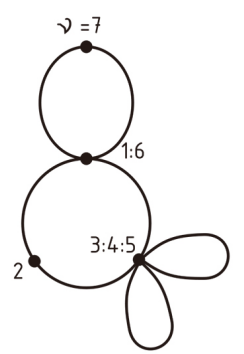

(50)-m

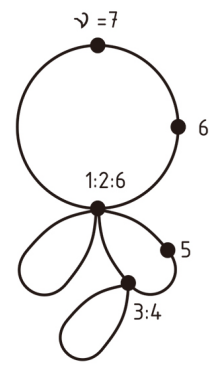

(51)-c

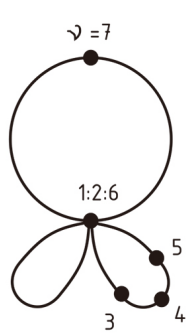

(51)

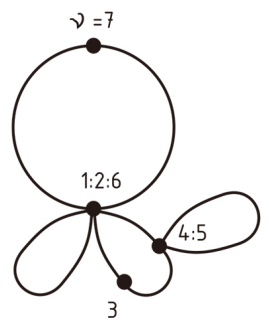

(51)-d

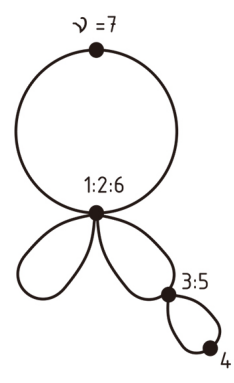

(51)-a

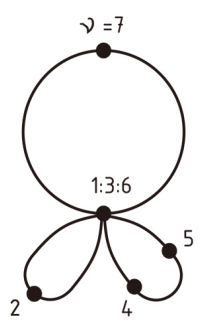

(52)

(a) 


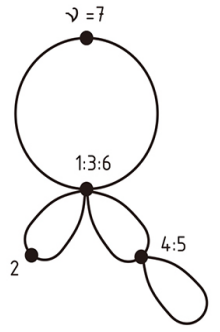

(52)-a

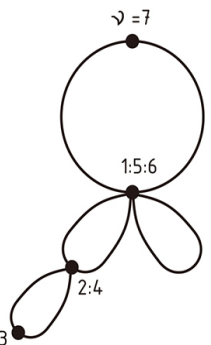

(54)-a

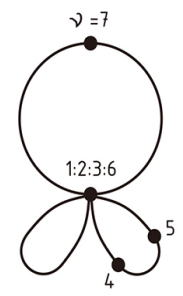

(55)

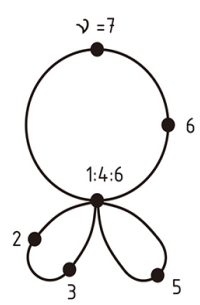

(53)

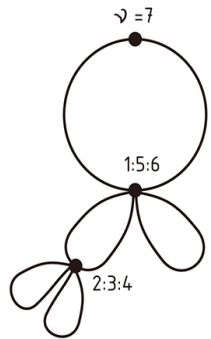

(54)-b

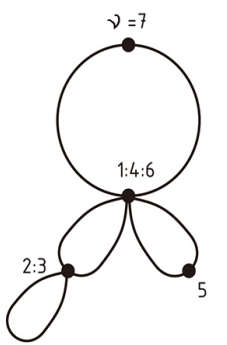

(53)-a

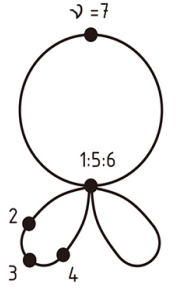

(54)

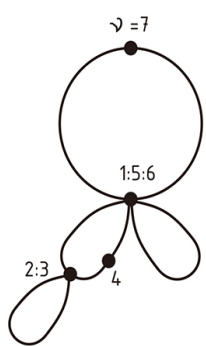

(54)-c

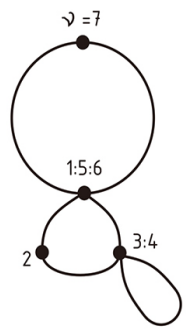

(54)-d

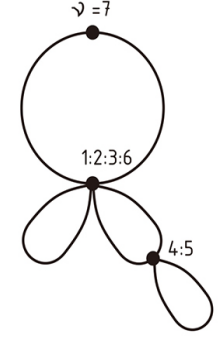

(55)-a

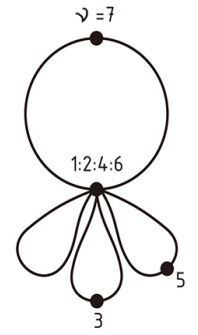

(56)

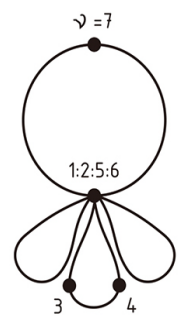

(57)

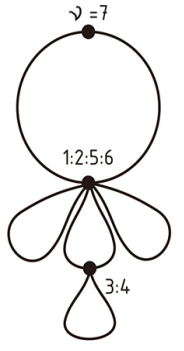

(57)-a

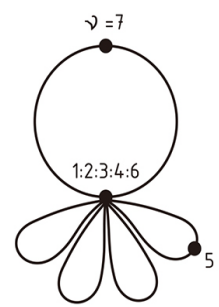

(61)

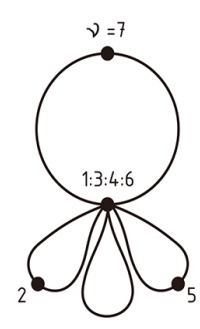

(58)

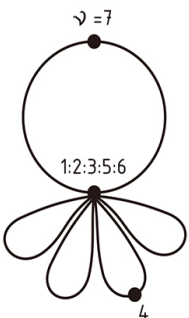

(62)

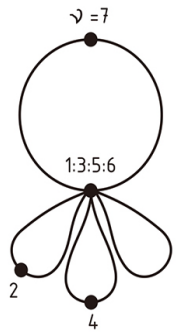

(59)

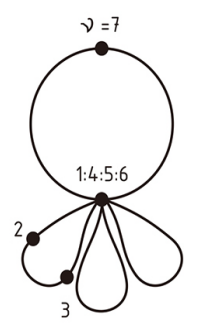

(60)

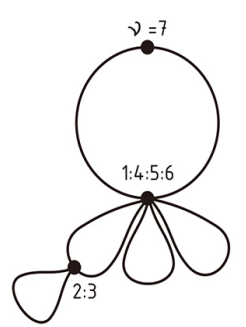

(60)-a

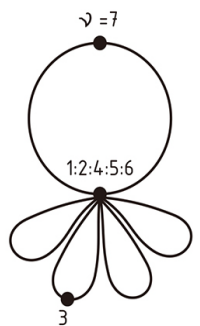

(63)

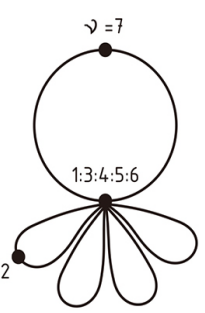

(64)

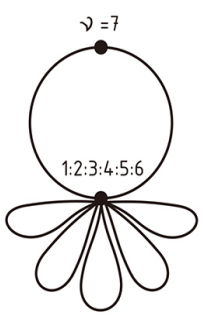

(65)

(b)

Figure 3. Diagrams representing the energy perturbation terms for $N=7$ obtained from contractions of the time points 1 and 6 , as well as contractions done together with the points between 1 and 6 . The numbers below diagrams refer to the formulae in the text. 


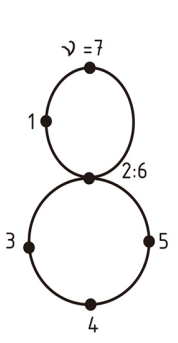

(69)

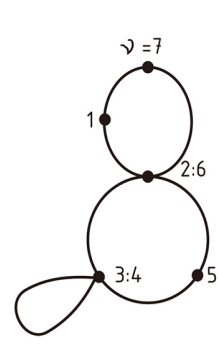

(69)-a

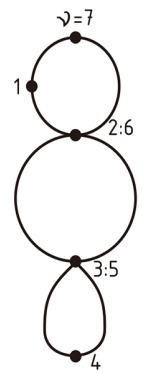

(69)-b

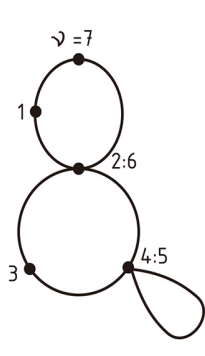

(69)-c

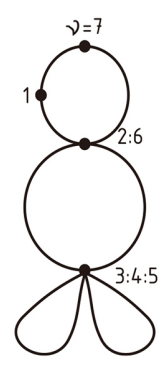

(69)-d

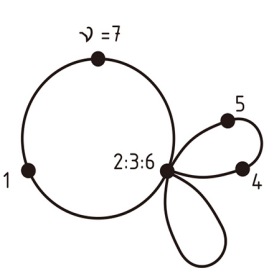

(70)

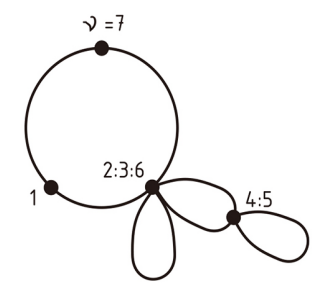

(70)-a

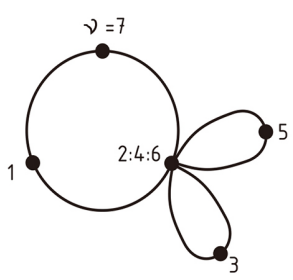

(71)

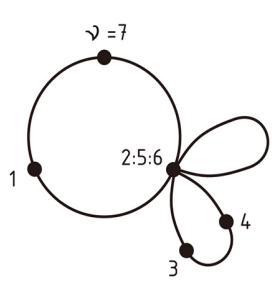

(72)

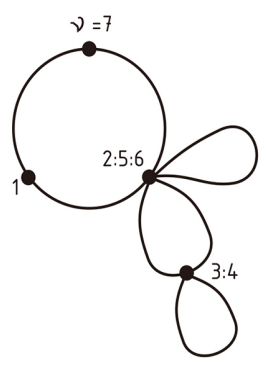

(72)-a

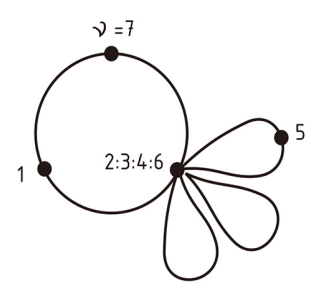

(73)

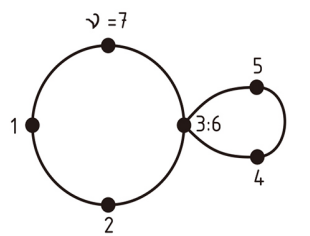

(81)

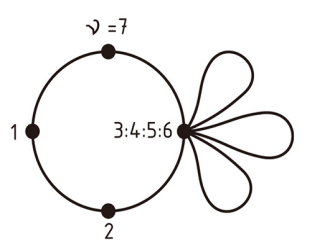

(84)

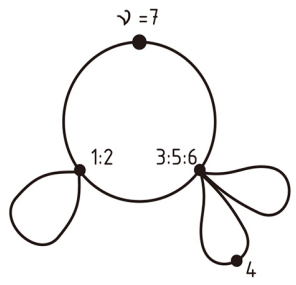

(89)

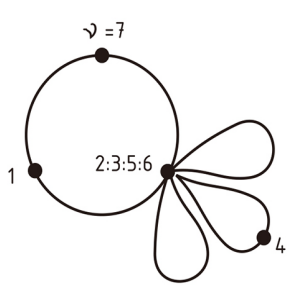

(74)

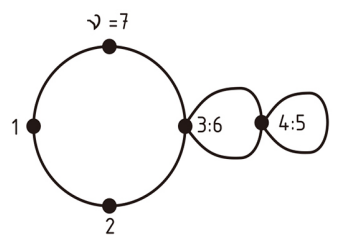

(81)-a

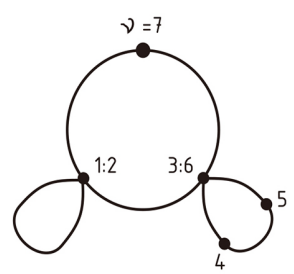

(87)

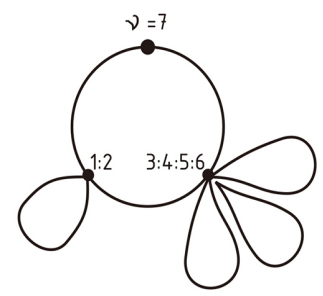

(90)

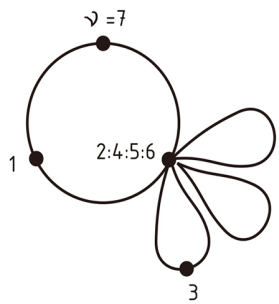

(75)

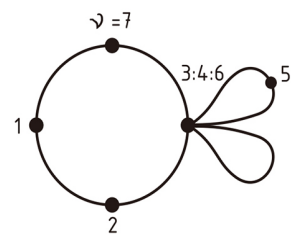

(82)

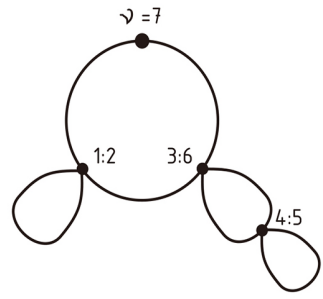

(87)-a

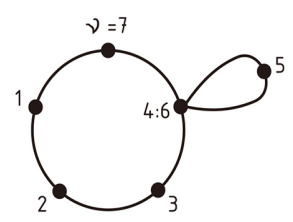

(96)

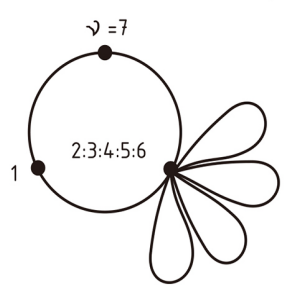

(76)

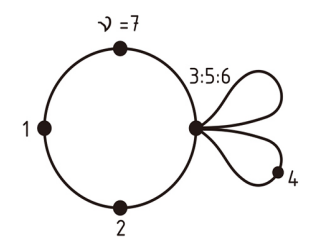

(83)

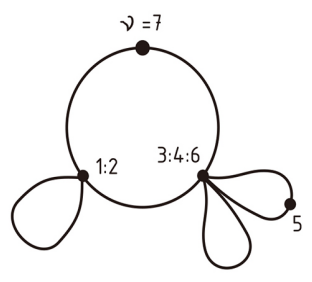

(88)

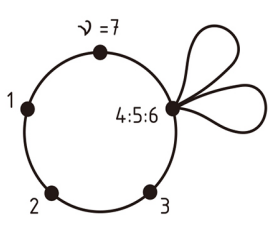

(97)

(a) 


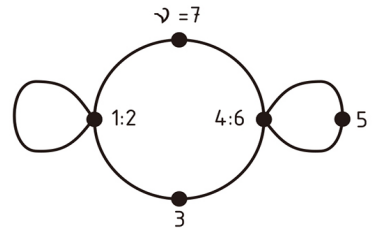

(98)

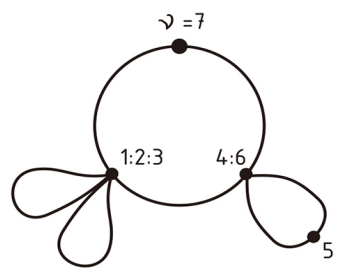

(102)

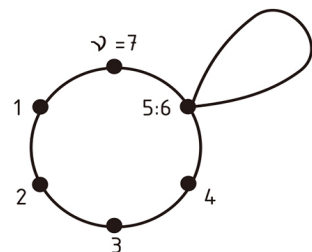

(110)

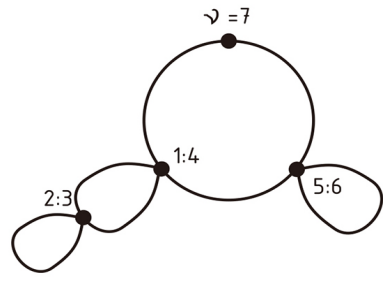

(113)-a

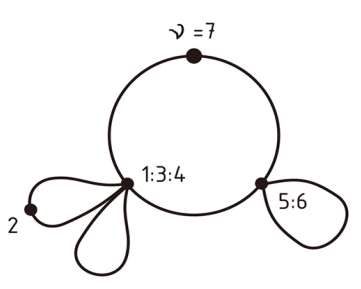

(117)

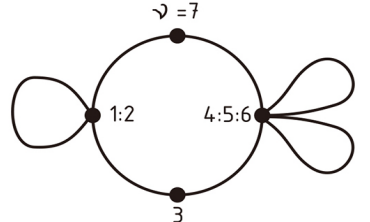

(99)

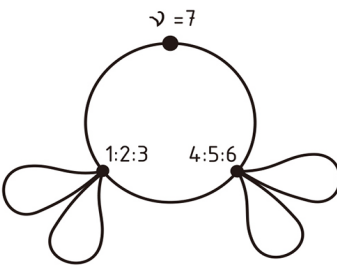

(103)

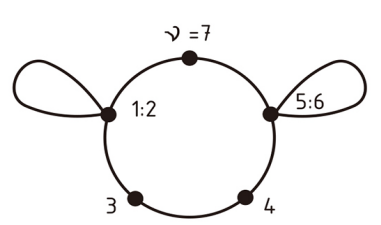

(111)

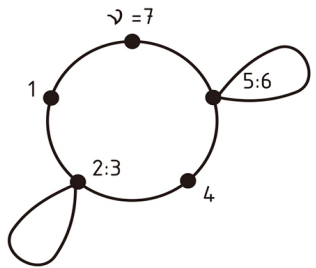

(114)

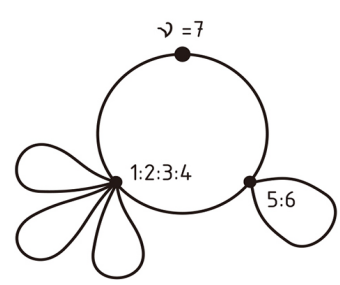

(118)

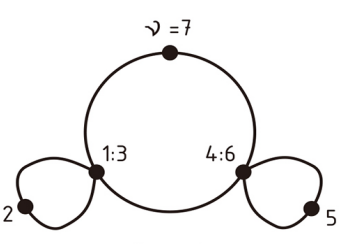

(100)

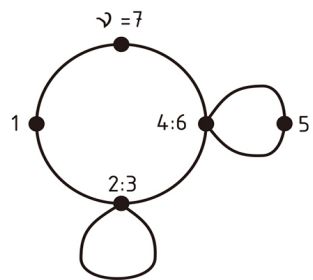

(104)

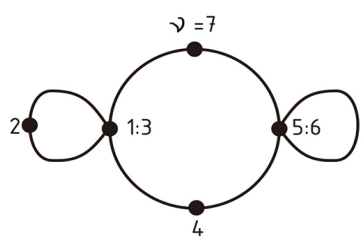

(112)

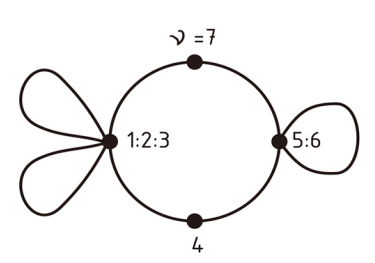

(115)

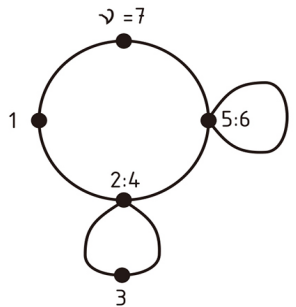

(119)

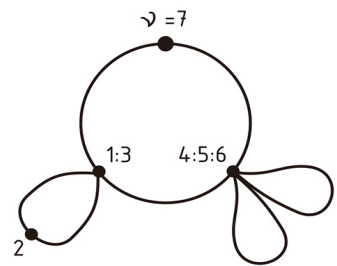

(101)

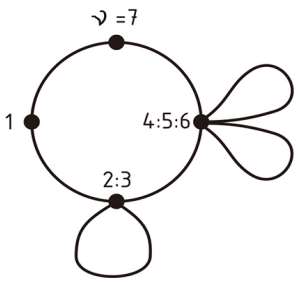

(105)

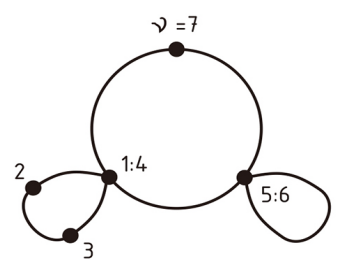

(113)

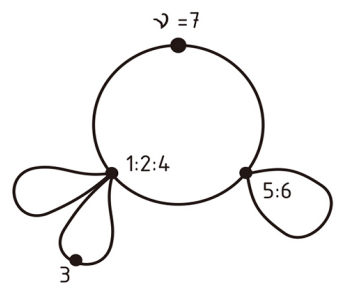

(116)

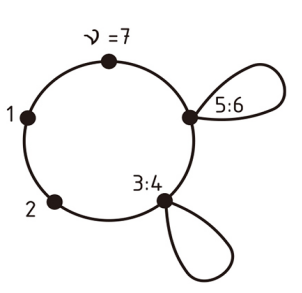

(120)

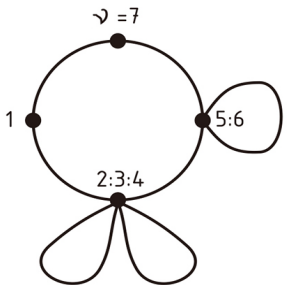

(121)

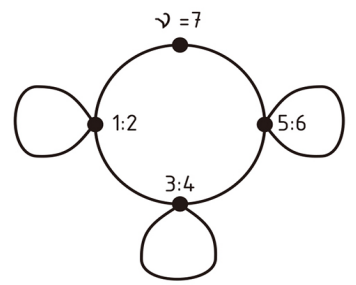

(122)

(b)

Figure 4. Diagrams representing the energy perturbation terms for $N=7$ which did not enter Figure 2 and Figure 3. Numbers below diagrams refer to the formulae presented in the text. 
If beyond of (69) we take into account also all possible contractions of the time points which are between 2 and 6 (see Figure 4), we obtain

$$
2: 3: 6 \rightarrow\left\langle V P V P^{3} V\right\rangle \Delta E_{1} \Delta E_{3}
$$

which contributes two energy terms,

$$
2: 4: 6 \rightarrow\left\langle V P V P^{3} V\right\rangle\left(\Delta E_{2}\right)^{2}
$$

which gives a single energy term,

$$
2: 5: 6 \rightarrow\left\langle V P V P^{3} V\right\rangle \Delta E_{3} \Delta E_{1}
$$

which is again a combination of two terms, and

$$
\begin{gathered}
2: 3: 4: 6 \rightarrow-\left\langle V P V P^{4} V\right\rangle\left(\Delta E_{1}\right)^{2} \Delta E_{2}, \\
2: 3: 5: 6 \rightarrow-\left\langle V P V P^{4} V\right\rangle \Delta E_{1} \Delta E_{2} \Delta E_{1}, \\
2: 4: 5: 6 \rightarrow-\left\langle V P V P^{4} V\right\rangle \Delta E_{2}\left(\Delta E_{1}\right)^{2}, \\
2: 3: 4: 5: 6 \rightarrow\left\langle V P V P^{5} V\right\rangle\left(\Delta E_{1}\right)^{4},
\end{gathered}
$$

which are all single energy terms. Together with contraction 2:6 in (69) all interactions containing points 2 and 6 [equations (69)-(76)] give the number of energy terms equal to

$$
5+2+1+2+1+1+1+1=14=S_{5} .
$$

In effect the lacking number of diagrams for $N=7$ is reduced to

$$
S_{7}-2 \times S_{6}-S_{5}=48-14=34 \text {. }
$$

A new "interaction" which is between points 3 and 6, symbolized by

$$
3: 6 \text {, }
$$

but involving also contractions with the points 4 and 5 placed between 3 and 6 , gives

$$
S_{4}=5
$$

new energy diagrams corresponding to contractions

$$
\begin{gathered}
3: 6 \rightarrow-\left\langle V P V P V P^{2} V\right\rangle \Delta E_{3}, \\
3: 4: 6 \rightarrow\left\langle V P V P V P^{3} V\right\rangle \Delta E_{1} \Delta E_{2}, \\
3: 5: 6 \rightarrow\left\langle V P V P V P^{3} V\right\rangle \Delta E_{2} \Delta E_{1}, \\
3: 4: 5: 6 \rightarrow-\left\langle V P V P V P^{4} V\right\rangle\left(\Delta E_{1}\right)^{3} .
\end{gathered}
$$

In fact the formulae (81)-(84) give

$$
2+1+1+1=5=S_{4}
$$

energy terms.

But this situation ignores a mutual relation between points 1 and 2 being outside contraction $3: 6$. This relation is represented by contraction of 1 and 2 given in (86) below. In effect we have two possibilities which have to be 
considered: one is when 1 and 2 remain free, but another one is when 1 and 2 "interact" in the form of contraction

$$
1: 2
$$

independently of the presence of (79) and (80). In result the number of diagrams represented by (80) should be taken twice: once it should be combined with free time points 1 and 2 [the terms (81)-(84)], otherwise it should be combined with the "interaction" of 1 and 2 given by contraction $1: 2$. In this second case the following 5 energy terms are obtained:

$$
\begin{gathered}
1: 2 \cap 3: 6 \rightarrow\left\langle V P^{2} V P^{2} V\right\rangle \Delta E_{1} \Delta E_{3}, \\
1: 2 \bigcap 3: 4: 6 \rightarrow-\left\langle V P^{2} V P^{3} V\right\rangle\left(\Delta E_{1}\right)^{2} \Delta E_{2}, \\
1: 2 \bigcap 3: 5: 6 \rightarrow-\left\langle V P^{2} V P^{3} V\right\rangle \Delta E_{1} \Delta E_{2} \Delta E_{1}, \\
1: 2 \bigcap 3: 4: 5: 6 \rightarrow\left\langle V P^{2} V P^{4} V\right\rangle\left(\Delta E_{1}\right)^{4},
\end{gathered}
$$

if we note that $\Delta E_{3}$ in (87) combines two terms.

The number of diagrams still necessary to calculate is

$$
S_{7}-2 \times S_{6}-S_{5}-2 \times S_{4}=34-10=24 \text {. }
$$

The last but one step is "interaction" of point 6 with point 4 , namely

$$
4: 6 \text {. }
$$

Since point 5 should not be isolated from the "interaction" with 4 and 6, still one contraction, namely

$$
4: 5: 6
$$

has to be considered together with (92). But because the points 1, 2, and 3 are remaining free beyond of 4 and 6 , the two energy diagrams which correspond respectively to (92) and (93) should combine with situations due to the presence of 1,2 , and 3. These points give $S_{4}=5$ cases:

$$
1,2,3 \text { are free }
$$

and four contractions of (94) which are

$$
1: 2,1: 3,1: 2: 3 \text { and } 2: 3 \text {. }
$$

The five situations given in (94) and (95) combined with two cases presented in (92) and (93) give in total

$$
2 \times S_{4}=10
$$

of new energy terms belonging to $N=7$. These are:

$$
\begin{gathered}
4: 6 \rightarrow-\left\langle V P V P V P V P^{2} V\right\rangle \Delta E_{2}, \\
4: 5: 6 \rightarrow\left\langle V P V P V P V P^{3} V\right\rangle\left(\Delta E_{1}\right)^{2}, \\
1: 2 \bigcap 4: 6 \rightarrow\left\langle V P^{2} V P V P^{2} V\right\rangle \Delta E_{1} \Delta E_{2}, \\
1: 2 \bigcap 4: 5: 6 \rightarrow-\left\langle V P^{2} V P V P^{3} V\right\rangle\left(\Delta E_{1}\right)^{3}, \\
1: 3 \cap 4: 6 \rightarrow\left\langle V P^{2} V P^{2} V\right\rangle\left(\Delta E_{2}\right)^{2},
\end{gathered}
$$




$$
\begin{gathered}
1: 3 \cap 4: 5: 6 \rightarrow-\left\langle V P^{2} V P^{3} V\right\rangle \Delta E_{2}\left(\Delta E_{1}\right)^{2}, \\
2: 3 \cap 4: 6 \rightarrow\left\langle V P V P^{2} V P^{2} V\right\rangle \Delta E_{1} \Delta E_{2}, \\
2: 3 \cap 4: 5: 6 \rightarrow-\left\langle V P V P^{2} V P^{3} V\right\rangle\left(\Delta E_{1}\right)^{3}, \\
1: 2: 3 \cap 4: 6 \rightarrow-\left\langle V P^{3} V P^{2} V\right\rangle\left(\Delta E_{1}\right)^{2} \Delta E_{2}, \\
1: 2: 3 \cap 4: 5: 6 \rightarrow\left\langle V P^{3} V P^{3} V\right\rangle\left(\Delta E_{1}\right)^{4} .
\end{gathered}
$$

The effect of (92)-(106) is reduction of the unknown terms to the number

$$
S_{7}-2 \times S_{6}-S_{5}-4 \times S_{4}=24-10=14 .
$$

But the number 14 in (107) can be obtained from a single contraction which remains to be considered, namely that between 6 and 5 :

$$
5: 6 \text {. }
$$

For in case of (108) four time points remain free on the scale: 1, 2, 3 and 4. Their combinations are:

$$
1,2,3 \text {, and } 4 \text { remain free }
$$

or the points give contractions:

$$
\begin{gathered}
1: 2, \quad 1: 3, \quad 1: 4, \quad 2: 3, \quad 2: 4, \quad 3: 4, \\
1: 2: 3, \quad 1: 2: 4, \quad 1: 3: 4, \quad 2: 3: 4, \\
1: 2: 3: 4, \quad 1: 2 \cap 3: 4, \quad 1: 4 \cap 2: 3 .
\end{gathered}
$$

The effect of (109) and (110) is that they give precisely $S_{5}=14$ configurations of the time points 1, 2, 3 and 4 necessary to construct the remainder of energy diagrams dictated by the result in (107). The energy terms due to (108)-(110) are:

$$
\begin{gathered}
5: 6 \rightarrow-\left\langle V P V P V P V P V P^{2} V\right\rangle \Delta E_{1}, \\
1: 2 \cap 5: 6 \rightarrow\left\langle V P^{2} V P V P V P^{2} V\right\rangle\left(\Delta E_{1}\right)^{2}, \\
1: 3 \cap 5: 6 \rightarrow\left\langle V P^{2} V P V P^{2} V\right\rangle \Delta E_{2} \Delta E_{1}, \\
1: 4 \cap 5: 6 \text { and } 1: 4 \cap 2: 3 \cap 5: 6 \rightarrow\left\langle V P^{2} V P^{2} V\right\rangle \Delta E_{3} \Delta E_{1},
\end{gathered}
$$

which is a combination of two energy terms,

$$
\begin{gathered}
2: 3 \cap 5: 6 \rightarrow\left\langle V P V P^{2} V P V P^{2} V\right\rangle\left(\Delta E_{1}\right)^{2}, \\
1: 2: 3 \cap 5: 6 \rightarrow-\left\langle V P^{3} V P V P^{2} V\right\rangle\left(\Delta E_{1}\right)^{3}, \\
1: 2: 4 \cap 5: 6 \rightarrow-\left\langle V P^{3} V P^{2} V\right\rangle \Delta E_{1} \Delta E_{2} \Delta E_{1}, \\
1: 3: 4 \cap 5: 6 \rightarrow-\left\langle V P^{3} V P^{2} V\right\rangle \Delta E_{2}\left(\Delta E_{1}\right)^{2}, \\
1: 2: 3: 4 \cap 5: 6 \rightarrow\left\langle V P^{4} V P^{2} V\right\rangle\left(\Delta E_{1}\right)^{4}, \\
2: 4 \cap 5: 6 \rightarrow\left\langle V P V P^{2} V P^{2} V\right\rangle \Delta E_{2} \Delta E_{1}, \\
3: 4 \cap 5: 6 \rightarrow\left\langle V P V P V P^{2} V P^{2} V\right\rangle\left(\Delta E_{1}\right)^{2},
\end{gathered}
$$




$$
\begin{gathered}
2: 3: 4 \cap 5: 6 \rightarrow-\left\langle V P V P^{3} V P^{2} V\right\rangle\left(\Delta E_{1}\right)^{3}, \\
1: 2 \cap 3: 4 \cap 5: 6 \rightarrow-\left\langle V P^{2} V P^{2} V P^{2} V\right\rangle\left(\Delta E_{1}\right)^{3} .
\end{gathered}
$$

In Table 2 we collect the Schrödinger terms belonging to $N=7$ which are due to the time contractions given below (78).

In effect the total value of the Schrödinger perturbation energy belonging to $N$ $=7$ is given by a sum of: (a) the terms present in Table 1 , (b) the terms given in formulae (50)-(65), (c) the terms in the formulae (69)-(76), (d) the terms collected in Table 2 .

In the next section we present the balance of the Huby-Tong number of the perturbation energy terms with the total number of energy diagrams obtainedfor a given $N$-from contractions of the time points on the circular scale.

\section{Balance of the Number of Perturbation Energy Terms Obtained from the Huby-Tong Formula and within the Framework of the Present Theory}

Let us take the perturbation orders $N=8,9$ and 10 for which the number of the Schrödinger perturbation terms calculated from the Huby-Tong formula [see (15)] is respectively equal to:

$$
\begin{gathered}
S_{8}=\frac{(2 \times 8-2) !}{8 ! 7 !}=429, \\
S_{9}=\frac{(2 \times 9-2) !}{9 ! 8 !}=1430, \\
S_{10}=\frac{(2 \times 10-2) !}{10 ! 9 !}=4862 .
\end{gathered}
$$

These results will be compared with the number of time diagrams obtained on the circular scale taken for the same $N$ as quoted above. The calculations performed with the aid of the circular scale are of a recurrent character which means that the knowledge of diagrams for $N-1, N-2$, etc., is used for calculation of the diagrams characteristic for $N$. The general rule is the same as presented in the preceding Section: we consider for a given $N$ the time scale characteristic by the presence of the number of $N-1$ time points suitable to contractions and add one time point to that ensemble.

Beginning with $N=8$ we have 7 time points "active" on the scale because the 8th point is the beginning-end point which cannot participate in contractions. The time point 7 is new for the "active" part of the scale for $N=7$ which had only 6 points of an active kind; see Section 6 . The presence of point 7 gives

$$
S_{7}=132
$$

new diagrams valid for $N=8$ on condition a modification of the diagrams energy by $P V$ present in formula (46) is taken into account.

The next set of $S_{7}$ diagrams participating in calculation of the terms belonging to $N=8$ is obtained from contraction 
Table 2. The 34 Schrödinger energy terms belonging to $N=7$ due to the time contractions presented below the formula (78). Each of expressions having $\Delta E_{3}$ combines two Schrödinger terms.

\begin{tabular}{|c|c|}
\hline$-\left\langle V P V P V P^{2} V\right\rangle \Delta E_{3}$ & [see (81)], \\
\hline$\left\langle V P V P V P^{3} V\right\rangle \Delta E_{1} \Delta E_{2}$ & [see $(82)]$ \\
\hline$\left\langle V P V P V P^{3} V\right\rangle \Delta E_{2} \Delta E_{1}$ & [see (83)], \\
\hline$-\left\langle V P V P V P^{2} V\right\rangle\left(\Delta E_{1}\right)^{3}$ & [see (84)], \\
\hline$\left\langle V P^{2} V P^{2} V\right\rangle \Delta E_{1} \Delta E_{3}$ & [see (87)], \\
\hline$-\left\langle V P^{2} V P^{3} V\right\rangle\left(\Delta E_{1}\right)^{2} \Delta E_{2}$ & [see (88)], \\
\hline$-\left\langle V P^{2} V P^{3} V\right\rangle \Delta E_{1} \Delta E_{2} \Delta E_{1}$ & [see (89)], \\
\hline$\left\langle V P^{2} V P^{4} V\right\rangle\left(\Delta E_{1}\right)^{4}$ & [see (90)], \\
\hline$-\left\langle V P V P V P V P^{2} V\right\rangle \Delta E_{2}$ & [see (97)], \\
\hline$\left\langle V P V P V P V P^{3} V\right\rangle\left(\Delta E_{1}\right)^{2}$ & [see (98)], \\
\hline$\left\langle V P^{2} V P V P^{2} V\right\rangle \Delta E_{1} \Delta E_{2}$ & [see (99)], \\
\hline$-\left\langle V P^{2} V P V P^{3} V\right\rangle\left(\Delta E_{1}\right)^{3}$ & {$[\operatorname{see}(100)]$, } \\
\hline$\left\langle V P^{2} V P^{2} V\right\rangle\left(\Delta E_{2}\right)^{2}$ & [see (101)], \\
\hline$-\left\langle V P^{2} V P^{3} V\right\rangle \Delta E_{2}\left(\Delta E_{1}\right)^{2}$ & [see (102)], \\
\hline$\left\langle V P V P^{2} V P^{2} V\right\rangle \Delta E_{1} \Delta E_{2}$ & [see (103)], \\
\hline$-\left\langle V P V P^{2} V P^{3} V\right\rangle\left(\Delta E_{1}\right)^{3}$ & [see (104)], \\
\hline$-\left\langle V P^{3} V P^{2} V\right\rangle\left(\Delta E_{1}\right)^{2} \Delta E_{2}$ & [see (105)], \\
\hline$\left\langle V P^{3} V P^{3} V\right\rangle\left(\Delta E_{1}\right)^{4}$ & [see (106)], \\
\hline$-\left\langle V P V P V P V P V P^{2} V\right\rangle \Delta E_{1}$ & [see (111)], \\
\hline$\left\langle V P^{2} V P V P V P^{2} V\right\rangle\left(\Delta E_{1}\right)^{2}$ & [see (112)], \\
\hline$\left\langle V P^{2} V P V P^{2} V\right\rangle \Delta E_{2} \Delta E_{1}$ & [see (113)], \\
\hline$\left\langle V P^{2} V P^{2} V\right\rangle \Delta E_{3} \Delta E_{1}$ & [see (114)], \\
\hline$\left\langle V P V P^{2} V P V P^{2} V\right\rangle\left(\Delta E_{1}\right)^{2}$ & {$[\operatorname{see}(115)]$} \\
\hline$-\left\langle V P^{3} V P V P^{2} V\right\rangle\left(\Delta E_{1}\right)^{3}$ & [see (116)], \\
\hline$-\left\langle V P^{3} V P^{2} V\right\rangle \Delta E_{1} \Delta E_{2} \Delta E_{1}$ & [see (117)], \\
\hline$-\left\langle V P^{3} V P^{2} V\right\rangle \Delta E_{2}\left(\Delta E_{1}\right)^{2}$ & [see (118)], \\
\hline$\left\langle V P^{4} V P^{2} V\right\rangle\left(\Delta E_{1}\right)^{4}$ & [see (119)], \\
\hline$\left\langle V P V P^{2} V P^{2} V\right\rangle \Delta E_{2} \Delta E_{1}$ & [see (120)], \\
\hline$\left\langle V P V P V P^{2} V P^{2} V\right\rangle\left(\Delta E_{1}\right)^{2}$ & [see (121)], \\
\hline$-\left\langle V P V P^{3} V P^{2} V\right\rangle\left(\Delta E_{1}\right)^{3}$ & [see (122)], \\
\hline$-\left\langle V P^{2} V P^{2} V P^{2} V\right\rangle\left(\Delta E_{1}\right)^{3}$ & {$[\operatorname{see}(123)]$. } \\
\hline
\end{tabular}

done together with contractions of the time points between 1 and 7 (namely 2, 3, 4,5 and 6 ).

Other components of $S_{8}$ are given by 


$$
S_{6}=42
$$

diagrams due to contraction 2:7 combined with points 3, 4, 5 and 6 enclosed between 2 and 7. Only one set of terms equal to (129) is obtained in this way because the only free time point 1 on the scale does not combine with any other time point.

The situation becomes different for contraction

$$
3: 7
$$

which-together with points 4,5 , and 6 - can give

$$
S_{5}=14
$$

diagrams for any arrangement of points 1 and 2. Since two such arrangements are possible (for contracted and free pair see Sec. 6), so in fact contraction (130) gives $2 S_{5}=28$ energy terms.

The contraction

$$
4: 7 \text {, }
$$

together with its associates, gives only $S_{4}=5$ terms but these terms apply to $S_{5}=5$ situations dictated by the arrangement of the time points 1,2 , and 3 . In effect $5 \times 5=25$ energy terms are obtained.

The remaining situations are given by contractions

$$
5: 7
$$

and

$$
6: 7
$$

The contraction in (133) has its associate in contraction

$$
5: 6: 7 \text {, }
$$

so two diagrams given by (133) and (133a) should be multiplied by

$$
S_{5}=14
$$

arrangements of the time points 1, 2, 3, and 4 which are outside of contractions (133) and (133a). This gives $2 \times 14$ new energy terms.

Finally a single contraction (134) corresponds to

$$
S_{6}=42
$$

arrangements of the points $1,2,3,4$, and 5 outside $6: 7$ giving the number of energy terms equal to $S_{6}$ in (135).

The total number of terms for $N=8$ due to contractions taken into account between the formulae (127) and (135) becomes:

$$
\begin{aligned}
& 2 \times S_{7}+S_{6}+2 \times S_{5}+S_{4} \times S_{4}+2 \times S_{5}+S_{6} \\
& =264+42+28+25+28+42=429
\end{aligned}
$$

which is the result equal to that given in (124). This implies a complete number of necessary contractions considered in the $N=8$ case.

Calculations similar to those for $N=8$ can be done for other $N$, too. The components entering them can be arranged in a more transparent way than in 
the case of (136); see Table 3 . The results fully agree with those obtained in (125), (126) and those calculated from (15).

\section{Summary}

The present paper considers the well-known Schrödinger perturbation series for energy of a non-degenerate quantum state; the applied perturbation potential does not depend on time.

A usual problem of the Schrödinger perturbation theory is that their formulae are derived in a tediously obtainable and complicated way. This concerns especially the case when a large order $N$ of the perturbation energy is examined. A difficulty concerns also the Schrödinger perturbation calculation developed with the aid of the Feynman diagrams. Here large $N$ imply a huge number of diagrams which have to be derived and considered in calculations; in effect the number of the Feynman diagrams can exceed by several orders of times the number of kinds of the perturbation terms entering the Schrödinger theory [3]. It should be noted that the scale of time applied by Feynman is a conventional scale extended from minus to plus infinity; see Section 2.

The paper demonstrates that a difficulty connected with construction of the Schrödinger perturbation terms can be overcomed with the aid of a circular scale of time. According to Leibniz, time is a successive sequence of events, or sets of events. In such a picture the time intervals between separate events, or their sets, play a secondary role. The history of a system is built up by following the development in time of the system configurations.

In case of the Schrödinger theory the time events are assumed to represent a gradual change of a quantum state upon the action of the perturbation potential. The events are successive collisions of the quantum system with that potential. The number of collisions is grouped in sets according to the size of the perturbation orders $N$ : the $N$ points of time are belonging to any set. These points are assumed to be arranged successively along a topological circle. In each set of $N$ points one of the points does represent the beginning-end point of the circular scale belonging to that $N$.

A result which seems to be important is that all kinds of the Schrödinger perturbation terms can be obtained-almost automatically, i.e. without calculations-from the arrangements of the time points present on the circle. To this purpose a special kind of interactions between the time points-called also contractions-should be assumed. A general rule concerning contractions is that the time loops created by them do not cross. In effect, the number of diagrams obtained due to contractions for a given $N$ agrees precisely with the number of kinds of the Schrödinger perturbation terms for that $N$.

The main aim of the paper became to present a recursive process to obtain all kinds of the Schrödinger perturbation terms belonging to a given $N$. This means we assume that the terms characteristic for $N-1, N-2, N-3, \cdots$ are known, and from them-and the points arrangement on the circular scale-all terms for $N$ can be obtained. The main feature of the process is to take properly into 
Table 3. Number of the perturbation terms for different $N$ obtained from the Huby-Tong formula in equation (15) compared with the terms number derived on the basis of the present theory; see also [17].

$$
\begin{array}{ll}
N=2: & S_{1} S_{1}=1=S_{2} \\
N=3: & S_{1} S_{2}+S_{2} S_{1}=1+1=2=S_{3} \\
N=4: & S_{1} S_{3}+S_{2} S_{2}+S_{3} S_{1}=2+1+2=5=S_{4} \\
N=5: & S_{1} S_{4}+S_{2} S_{3}+S_{3} S_{2}+S_{4} S_{1}=5+2+2+5=14=S_{5} \\
N=6: & S_{1} S_{5}+S_{2} S_{4}+S_{3} S_{3}+S_{4} S_{2}+S_{5} S_{1}=14+5+4+5+14=42=S_{6} \\
& S_{1} S_{6}+S_{2} S_{5}+S_{3} S_{4}+S_{4} S_{3}+S_{5} S_{2}+S_{6} S_{1} \\
N=7: & =42+14+10+10+14+42=132=S_{7} \\
& S_{1} S_{7}+S_{2} S_{6}+S_{3} S_{5}+S_{4} S_{4}+S_{5} S_{3}+S_{6} S_{2}+S_{1} S_{1} \\
N=8: & =2 \times(132+42+28)+25=429=S_{8} \\
N=9: & S_{1} S_{8}+S_{2} S_{7}+S_{3} S_{6}+S_{4} S_{5}+S_{5} S_{4}+S_{6} S_{3}+S_{7} S_{2}+S_{8} S_{1} \\
& =2 \times(429+132+84+70)=2 \times 715=1430=S_{9} \\
N=10: & S_{1} S_{9}+S_{2} S_{8}+S_{3} S_{7}+S_{4} S_{6}+S_{5} S_{5}+S_{6} S_{4}+S_{7} S_{3}+S_{8} S_{2}+S_{9} S_{1} \\
& =2 \times(1430+429+264+210)+196=4862=S_{10}
\end{array}
$$

account the fact that any time point present on the scale-beyond of the beginning-end point-should "interact" with other time points on that scale in a way characteristic for the contraction properties possessed by the time points on the scale.

In result we find that a tedious process of solving the perturbed Schrödinger equation-established in an ordinary three-dimensional space-can be replaced, with the aid of a new scale of time, by a very simple calculation of the Schrödinger energy solution.

\section{References}

[1] Schrödinger, E. (1926) Annalen der Physik, 80, 437. https://doi.org/10.1002/andp.19263851302

[2] Schiff, L.I. (1955) Quantum Mechanics. 2nd Edition, McGraw-Hill, New York.

[3] Mattuck, R.D. (1976) A Guide to Feynman Diagrams in a Many-Body Problem. 2nd Edition, McGraw-Hill, New York.

[4] Feynman, R.P. (1966) Science, 153, 699. https://doi.org/10.1126/science.153.3737.699

[5] Leibniz, G.W. (1924) Hauptschriften zur Grundlagen der Philosophie. Vol. 1, Leipzig.

[6] Rescher, N. (2013) On Leibniz. University of Pittsburgh Press. https://doi.org/10.2307/j.ctt7zw8g2

[7] Olszewski, S. (1991) Zeitschrift fur Naturforschung, 46A, 313.

[8] Olszewski, S. and Kwiatkowski, T. (1998) Computers in Chemistry, 22, 445. https://doi.org/10.1016/S0097-8485(98)00023-0

[9] Olszewski, S. (2003) Trends in Physical Chemistry, 9, 69.

[10] Olszewski, S. (2013) Quantum Matter, 2, 481. https://doi.org/10.1166/qm.2013.1085

[11] Olszewski, S. (2014) Journal of Modern Physics, 5, 1502; 
Olszewski, S. (2017) Journal of Modern Physics, 8, 1650.

[12] Olszewski, S. (2014) Journal of Quantum Information Science, 4, 269. https://doi.org/10.4236/jqis.2014.44022

[13] Olszewski, S. (2015) Quantum Matter, 4, 5523.

[14] Olszewski, S. (2004) Studia Philosophae Christianae, 40, 57.

[15] Huby, R. (1961) Proceedings of the Physical Society(London), 78, 529. https://doi.org/10.1088/0370-1328/78/4/306

[16] Tong, B.Y. (1962) Proceedings of the Physical Society (London), 80, 1101. https://doi.org/10.1088/0370-1328/80/5/308

[17] Olszewski, S. (2011) Journal of Quantum Information Science, 1, 142. https://doi.org/10.4236/jqis.2011.13020 


\section{Appendix: Perturbation Energy Calculation Due to the} Present Method Outlined for Low $N$ (from $N=1$ to $N=6$ )

We begin with the result that for $N=1$ the perturbation energy is

$$
\Delta E_{1}=\langle V\rangle=\left\langle n\left|V^{\text {per }}\right| n\right\rangle,
$$

and the loop of time is a topological circle with a single time point on it [7]-[14]. Consequently to the rule outlined in the present paper [see (46)] for $N=2$ we have

$$
\Delta E_{2}=\langle V P V\rangle
$$

The presence of a single $P$ in (2) indicates a single infinite summation over the unperturbed quantum states with exclusion of the perturbed state $n$; see (28) and (29). The time scale-beyond of the beginning-end point-has only one point on it [7]-[14].

The first step for $N=3$ is to substitute $P V$ at the end of the expression on the right of (A2). We obtain the first (positive) term for $\Delta E_{3}$ which is a double sum over the quantum states:

$$
\langle V P V P V\rangle
$$

But beyond of the beginning-end point on the scale we have still two free time points on it, say 1 and 2 . They should contract together giving the next term of $\Delta E_{3}$ equal to

$$
-\left\langle V P^{2} V\right\rangle\langle V\rangle
$$

this term is a single sum over the unperturbed states. The $\Delta E_{3}$ is

$$
\Delta E_{3}=\langle V P V P V\rangle-\left\langle V P^{2} V\right\rangle\langle V\rangle \text {. }
$$

In order to calculate the first two terms for $N=4$ we increase the term in (A3) again by $P V$ at the brackets end, and the same we are doing with the larger bracket term in (A4). We obtain two terms belonging to $\Delta E_{4}$ :

$$
\langle V P V P V P V\rangle
$$

and

$$
-\left\langle V P^{2} V P V\right\rangle\langle V\rangle=-\left\langle V P^{2} V P V\right\rangle \Delta E_{1}
$$

A suplementary point 3 which comes for $N=4$ can contract with points 1 and 2 entering the scale already for $N=3$. The interaction between 3 and 1 gives two contractions:

$$
1: 3, \quad 1: 2: 3
$$

whereas the interaction between 3 and 2 alone is reduced to contraction

$$
2: 3 \text {. }
$$

The energy terms representing (A8) are respectively

$$
-\left\langle V P^{2} V\right\rangle\langle V P V\rangle=-\left\langle V P^{2} V\right\rangle \Delta E_{2}
$$

and

$$
\left\langle V P^{3} V\right\rangle\langle V\rangle\langle V\rangle=\left\langle V P^{3} V\right\rangle\left(\Delta E_{1}\right)^{2}
$$

and the term represented by (A9) is 


$$
-\left\langle V P V P^{2} V\right\rangle\langle V\rangle=-\left\langle V P V P^{2} V\right\rangle \Delta E_{1}
$$

Terms (A6), (A7) and (A10)-(A12) give together $S_{4}=5$ terms which is the number of the Schrödinger energy terms predicted by Huby and Tong [15] [16]. The sum of these terms gives:

$$
\begin{aligned}
\Delta E_{4}= & \langle V P V P V P V\rangle-\left\langle V P^{2} V P V\right\rangle \Delta E_{1}-\left\langle V P^{2} V\right\rangle \Delta E_{2} \\
& +\left\langle V P^{3} V\right\rangle\left(\Delta E_{1}\right)^{2}-\left\langle V P V P^{2} V\right\rangle \Delta E_{1} .
\end{aligned}
$$

The first five Schrödinger terms belonging to $\Delta E_{5}$ are given by modification of (A13). These are:

$$
\begin{aligned}
& \langle V P V P V P V P V\rangle,-\left\langle V P^{2} V P V P V\right\rangle \Delta E_{1},-\left\langle V P^{2} V P V\right\rangle \Delta E_{2}, \\
& \left\langle V P^{3} V P V\right\rangle\left(\Delta E_{1}\right)^{2} \text { and }\left\langle-V P V P^{2} V P V\right\rangle \Delta E_{1} .
\end{aligned}
$$

The remaining nine terms of $\Delta E_{5}$ come, first, from five contractions of a supplementary point 4 with point 1 and points between 1 and 4 :

$$
1: 4, \quad 1: 2: 4, \quad 1: 3: 4, \quad 1: 2: 3: 4, \quad 1: 4 \cap 2: 3 .
$$

They give respectively the five terms

$$
-\left\langle V P^{2} V\right\rangle \Delta E_{3},\left\langle V P^{3} V\right\rangle \Delta E_{1} \Delta E_{2},\left\langle V P^{3} V\right\rangle \Delta E_{2} \Delta E_{1},-\left\langle V P^{4} V\right\rangle\left(\Delta E_{1}\right)^{3},
$$

since the first term is due to combination of 2 terms, namely these given by the first and last contraction expression in (A15).

Other Schrödinger terms are due to contraction between points 2 and 4:

$$
2: 4, \quad 2: 3: 4
$$

and between points

$$
3: 4
$$

The last contraction leaves points 1 and 2 as free to contract together, so (A17) gives in fact two kinds of the Schrödinger terms: one term for uncontracted 1 and 2 , and one for the contracted case. In effect we have four terms belonging to $\Delta E_{5}$ coming from (A16) and (A17):

$$
\begin{array}{r}
-\left\langle V P V P^{2} V\right\rangle \Delta E_{2},\left\langle V P V P^{3} V\right\rangle\left(\Delta E_{1}\right)^{2}, \\
-\left\langle V P V P V P^{2} V\right\rangle \Delta E_{1},\left\langle V P^{2} V P^{2} V\right\rangle\left(\Delta E_{1}\right)^{2},
\end{array}
$$

where the last term is due to contraction $1: 2 \bigcap 3: 4$.

The sum of results obtained in (A14)-(A18) gives the 5th order perturbation energy combined by 14 Schrodinger terms:

$$
\begin{aligned}
\Delta E_{5}= & \langle V P V P V P V P V\rangle-\left\langle V P^{2} V P V P V\right\rangle \Delta E_{1}-\left\langle V P^{2} V P V\right\rangle \Delta E_{2} \\
& +\left\langle V P^{3} V P V\right\rangle\left(\Delta E_{1}\right)^{2}-\left\langle V P V P^{2} V P V\right\rangle \Delta E_{1} \\
& -\left\langle V P^{2} V\right\rangle \Delta E_{3}+\left\langle V P^{3} V\right\rangle \Delta E_{1} \Delta E_{2}+\left\langle V P^{3} V\right\rangle \Delta E_{2} \Delta E_{1} \\
& -\left\langle V P^{4} V\right\rangle\left(\Delta E_{1}\right)^{3}-\left\langle V P V P^{2} V\right\rangle \Delta E_{2}+\left\langle V P V P^{3} V\right\rangle\left(\Delta E_{1}\right)^{2} \\
& -\left\langle V P V P V P^{2} V\right\rangle \Delta E_{1}+\left\langle V P^{2} V P^{2} V\right\rangle\left(\Delta E_{1}\right)^{2} .
\end{aligned}
$$


The last perturbation order of energy considered in Appendix is $N=6$. In the first step we obtain 14 components of $\Delta E_{6}$ by modifying the energy components of $\Delta E_{5}$. They are obtained by adding $P V$ at the end of the main brackets term:

$$
\begin{gathered}
\langle V P V P V P V P V P V\rangle, \\
-\left\langle V P^{2} V P V P V P V\right\rangle\langle V\rangle=-\left\langle V P^{2} V P V P V P V\right\rangle \Delta E_{1}, \\
-\left\langle V P^{2} V P V P V\right\rangle\langle V P V\rangle=-\left\langle V P^{2} V P V P V\right\rangle \Delta E_{2}, \\
\left\langle V P^{3} V P V P V\right\rangle(\langle V\rangle)^{2}=\left\langle V P^{3} V P V P V\right\rangle\left(\Delta E_{1}\right)^{2}, \\
-\left\langle V P V P^{2} V P V P V\right\rangle\langle V\rangle=-\left\langle V P V P^{2} V P V P V\right\rangle \Delta E_{1}, \\
-\left\langle V P^{2} V P V\right\rangle \Delta E_{3}, \\
\left\langle V P^{3} V P V\right\rangle \Delta E_{1} \Delta E_{2}, \\
\left\langle V P^{3} V P V\right\rangle \Delta E_{2} \Delta E_{1}, \\
-\left\langle V P^{4} V P V\right\rangle\left(\Delta E_{1}\right)^{3}, \\
-\left\langle V P V P^{2} V P V\right\rangle \Delta E_{2}, \\
\left\langle V P V P^{3} V P V\right\rangle\left(\Delta E_{1}\right)^{2}, \\
-\left\langle V P V P V P^{2} V P V\right\rangle \Delta E_{1} \\
\left\langle V P^{2} V P^{2} V P V\right\rangle\left(\Delta E_{1}\right)^{2} .
\end{gathered}
$$

The term having $\Delta E_{3}$ as a multiplier combines two Schrödinger terms.

The next 14 terms belonging to $\Delta E_{6}$ are different than (A20); they are:

$$
\begin{gathered}
1: 5 \rightarrow-\left\langle V P^{2} V\right\rangle \Delta E_{4}, \\
1: 2: 5 \rightarrow\left\langle V P^{3} V\right\rangle \Delta E_{1} \Delta E_{3}, \\
1: 3: 5 \rightarrow\left\langle V P^{3} V\right\rangle\left(\Delta E_{2}\right)^{2}, \\
1: 4: 5 \rightarrow\left\langle V P^{3} V\right\rangle \Delta E_{3} \Delta E_{1}, \\
1: 2: 3: 5 \rightarrow-\left\langle V P^{4} V\right\rangle\left(\Delta E_{1}\right)^{2} \Delta E_{2}, \\
1: 2: 4: 5 \rightarrow-\left\langle V P^{4} V\right\rangle \Delta E_{1} \Delta E_{2} \Delta E_{1}, \\
1: 3: 4: 5 \rightarrow-\left\langle V P^{4} V\right\rangle \Delta E_{2}\left(\Delta E_{1}\right)^{2}, \\
1: 2: 3: 4: 5 \rightarrow\left\langle V P^{5} V\right\rangle\left(\Delta E_{1}\right)^{4} .
\end{gathered}
$$

The $\Delta E_{4}$ combines 5 Schrödinger terms and $\Delta E_{3}$ combines 2 terms. On the other hand, the last 14 terms of $\Delta E_{6}$ combine with the terms of (A20):

$$
\begin{gathered}
2: 5 \rightarrow-\left\langle V P V P^{2} V\right\rangle \Delta E_{3}, \\
2: 3: 5 \rightarrow\left\langle V P V P^{3} V\right\rangle \Delta E_{1} \Delta E_{2},
\end{gathered}
$$




$$
\begin{gathered}
2: 4: 5 \rightarrow\left\langle V P V P^{3} V\right\rangle \Delta E_{2} \Delta E_{1}, \\
2: 3: 4: 5 \rightarrow-\left\langle V P V P^{4} V\right\rangle\left(\Delta E_{1}\right)^{3}, \\
3: 5 \rightarrow-\left\langle V P V P V P^{2} V\right\rangle \Delta E_{2}, \\
3: 4: 5 \rightarrow\left\langle V P V P V P^{3} V\right\rangle\left(\Delta E_{1}\right)^{2}, \\
1: 2 \bigcap 3: 5 \rightarrow\left\langle V P^{2} V P^{2} V\right\rangle \Delta E_{1} \Delta E_{2}, \\
1: 2 \bigcap 3: 4: 5 \rightarrow-\left\langle V P^{2} V P^{3} V\right\rangle\left(\Delta E_{1}\right)^{3}, \\
4: 5 \rightarrow-\left\langle V P V P V P V P^{2} V\right\rangle \Delta E_{1}, \\
1: 2 \cap 4: 5 \rightarrow\left\langle V P^{2} V P V P^{2} V\right\rangle\left(\Delta E_{1}\right)^{2}, \\
1: 3 \cap 4: 5 \rightarrow-\left\langle V P^{2} V P^{2} V\right\rangle \Delta E_{2} \Delta E_{1}, \\
1: 2: 3 \cap 4: 5 \rightarrow-\left\langle V P^{3} V P^{2} V\right\rangle\left(\Delta E_{1}\right)^{3}, \\
2: 3 \cap 4: 5 \rightarrow\left\langle V P V P^{2} V P^{2} V\right\rangle\left(\Delta E_{1}\right)^{2} .
\end{gathered}
$$

In total we obtain for $N=6$ from (A20)-(A22) the number of terms

$$
14+14+14=42=S_{6} \text {. }
$$

The perturbation energy $\Delta E_{6}$ is equal to a sum of the terms presented in (A20)-(A22). 